\title{
Eliminating Impulse for Descriptor System by Derivative Output Feedback
}

\author{
Jian Li, ${ }^{1}$ Yufa Teng, ${ }^{2}$ Qingling Zhang, ${ }^{1}$ Jinghao Li, ${ }^{1}$ and Liang Qiao ${ }^{1}$ \\ ${ }^{1}$ Institute of Systems Science, Northeastern University, Shenyang, Liaoning 110819, China \\ ${ }^{2}$ Department of Mathematics, Air Force Logistics College, Xuzhou, Jiangsu 221000, China \\ Correspondence should be addressed to Qingling Zhang; qlzhang@mail.neu.edu.cn
}

Received 20 November 2013; Revised 6 February 2014; Accepted 7 February 2014; Published 20 May 2014

Academic Editor: Mark A. Petersen

Copyright (c) 2014 Jian Li et al. This is an open access article distributed under the Creative Commons Attribution License, which permits unrestricted use, distribution, and reproduction in any medium, provided the original work is properly cited.

\begin{abstract}
The problem of impulse elimination for descriptor system by derivative output feedback is investigated in this paper. Based on a novelly restricted system equivalence between matrix pencils, the range of dynamical order of the resultant closed loop descriptor system is given. Then, for the different dynamical order, sufficient conditions for the existence of derivative output feedback to ensure the resultant closed loop system to be impulse free are derived, and the corresponding derivative output feedback controllers are provided. Finally, simulation examples are given to show the consistence with the theoretical results obtained in this paper.
\end{abstract}

\section{Introduction}

Descriptor systems are also referred to as singular systems, implicit systems, differential algebraic systems, semistate systems, or generalized state space systems. Descriptor systems' models are more convenient and natural than standard state space systems' models in describing practical systems, such as interconnected large-scale systems, economic systems, networks, power systems, and biological systems $[1,2]$. Due to this reason, the control of descriptor systems has been extensively studied in past years and a great number of results based on the theory of standard state space systems have been generalized to descriptor systems [1-5].

The main feature of descriptor systems is regularity and impulsive behavior, which is different from standard state space systems. The regularity guarantees the existence and uniqueness of state responses of descriptor systems. The impulsive behavior may cause degradation in performance or even destroy the systems. Therefore, it is essentially important to use feedback control to eliminate the impulse for descriptor systems. Recently, robust impulse elimination control problems for uncertain descriptor system were investigated in [6], where it is established that an arbitrarily large impulse margin may be specified by output feedback if and only if the descriptor system is both controllable and observable at infinity in the sense of Rosenbrock. In some cases, derivative feedback is able to eliminate the impulse while the proportional feedback fails to do it. Now let us take an example to illustrate this fact; a three-dimensional descriptor system is given by

$$
E \dot{x}=A x+B u, \quad y=C x,
$$

where

$$
\begin{array}{cc}
E=\left[\begin{array}{lll}
1 & 0 & 0 \\
0 & 0 & 1 \\
0 & 0 & 0
\end{array}\right], \quad A=\left[\begin{array}{ccc}
-1 & 0 & 0 \\
0 & 1 & 0 \\
0 & 0 & 1
\end{array}\right], \\
B=\left[\begin{array}{l}
0 \\
1 \\
0
\end{array}\right], \quad C=\left[\begin{array}{lll}
0 & 0 & 1
\end{array}\right] .
\end{array}
$$

The system possesses impulse behavior and is not impulsive controllable. Obviously, the impulse will not be eliminated by any proportional feedback. However, one can choose derivative output feedback $u=\dot{y}$ to eliminate the impulse. In this case, the resultant closed loop system turns to be

$$
\left[\begin{array}{lll}
1 & 0 & 0 \\
0 & 0 & 0 \\
0 & 0 & 0
\end{array}\right] \dot{x}=\left[\begin{array}{ccc}
-1 & 0 & 0 \\
0 & 1 & 0 \\
0 & 0 & 1
\end{array}\right] x .
$$


It is easily seen that the resultant closed loop system is impulse free; that is, the impulse is eliminated by the derivative output feedback.

On the other hand, in some practical control, it is convenient to use derivative feedback. For instance, in controlling the suppression of vibration in mechanical systems, the derivative state signals are easier to obtain than the proportional state signals. Because derivative coefficient matrix $E$ can only be altered by derivative feedback, derivative feedback has an advantage over the proportional feedback in certain status of both theoretical analysis and practical application. It is especially effective to use derivative feedback in control of descriptor systems.

Up to now, lots of results regarding derivative feedback have been proposed. A derivative feedback controller for standard state space systems was designed via linear matrix inequalities technique to make the resultant closed loop system stable in [7]. Pole placement problems of the systems were discussed by derivative state feedback in [810]. The regularization of descriptor systems was discussed via derivative feedback and proportional plus derivative state feedback, respectively, in [11-13]. Robust derivative state feedback LMI-based designs for linear time invariant systems were recently proposed in [14]. Dynamical order assignment for linear descriptor systems was discussed in [15]. Using proportional plus derivative state feedback and proportional plus derivative output feedback, the normalization and stabilization problems for descriptor systems were investigated in [16]. Robust normalization and guaranteed cost control for a class of uncertain descriptor systems were investigated in [17]. Robust derivative state feedback for linear descriptor systems was studied through LMIs in [18]. Sufficient conditions for existence of derivative state feedback controllers were proposed in [19] to guarantee the resultant closed loop systems to be impulse free from minimum to maximum dynamical order. Based on orthogonal matrix decomposition, proportional and derivative state feedbacks were constructed in [20] such that the resultant closed loop systems are impulse free under various controllability conditions. On the basis of condensed forms, several necessary and sufficient conditions were derived to ensure the resultant closed loop system to be regular and impulse free by proportional and derivative output feedback [21-24], and the corresponding proportional and derivative output feedbacks can be resolved in a numerically stable way.

As we mentioned above, in some cases, elimination of the impulse will not be realized by proportional feedback, but it could be realized by derivative feedback under some conditions. It should be pointed out that derivative feedback will eliminate the impulse if proportional feedback can eliminate it. Although eliminating impulse by proportional and derivative output feedback has been investigated by [13, 20-24], the matrix factorizations used in these papers are complex and creative; this paper aims to address the effect of derivative output feedback on the impulsiveness alone and provide a tractable and alternative method to design the derivative output feedback. Motivated by the above analysis, the problems of eliminating the impulse of descriptor system by derivative output feedback are investigated in this paper. Combined with the advantage of dynamic model in overcoming the uncertain factors and disturbances, the descriptor systems with arbitrarily possibly dynamical order are investigated, and the sufficient conditions are established by which the resultant closed loop systems with any possibly dynamical order are impulse free; then the relevant derivative output feedback controllers are designed. The design method is theoretically effective and practically applicable.

This paper is structured as follows. In Section 2, some notations, definitions, and lemmas are recalled for later use. Section 3 gives the minimum rank $\tau_{\min }$ and related theorems to describe it. In Section 4, according to the different scope of the possibly dynamical order, some theorems by which the derivative output feedback controllers can be designed are proposed to ensure the resultant closed loop system to be impulse free. The effectiveness and merits of the main results are illustrated in Section 5 through several examples. Finally, remarking conclusion is made in Section 6.

\section{Preliminaries}

Consider a linear time invariant descriptor system of the following form:

$$
\begin{gathered}
E \dot{x}(t)=A x(t)+B u(t), \\
y(t)=C x(t)
\end{gathered}
$$

where $x(t) \in \mathbb{R}^{n}$ is state vector, $u(t) \in \mathbb{R}^{m}$ is control input, and $y(t) \in \mathbb{R}^{l}$ is controlled output, $E \in \mathbb{R}^{n \times n}$ is singular matrix with $\operatorname{rank} E=n_{1} \leq n$, and $B \in \mathbb{R}^{n \times m}, C \in \mathbb{R}^{l \times n}$ are full column rank and full row rank, respectively. System (4) is assumed to be regular; that is, $\operatorname{det}(s E-A) \neq 0$. In this case, the state response of system (4) exists uniquely for any admissible initial state.

As we know, the rank of derivative coefficient matrix $E$ equals the dynamical order of the system. To improve the system performance, it is often required to change the dynamical order, that is, the rank of matrix $E$, which can only be done by derivative feedback under certain conditions. It is also mentioned in the above section that the derivative feedback is an efficient tool to eliminate the impulse. Therefore, the main purpose of this paper is to use derivative output feedback to eliminate the impulse and make the resultant closed loop system regular.

To this end, the derivative output feedback in a general form can be described as

$$
u(t)=-L \dot{y}(t)+v(t)
$$

where $L \in \mathbb{R}^{m \times l}$ is the gain matrix to be determined and $v(t)$ is the new control input with appropriate dimension.

Applying the feedback controller (5) to system (4), the resultant closed loop system is obtained as

$$
(E+B L C) \dot{x}(t)=A x(t)+B v(t) \text {. }
$$

Consequently, our task now is to establish the criteria to ensure the resultant closed loop system to be impulse free 
with arbitrarily possibly dynamical order. To fulfil our task, we need the following statement and several lemmas.

For system (4), it is easy to prove that there exist four nonsingular matrices $\bar{P}, \bar{Q}, \widetilde{P}$, and $\widetilde{Q}$ such that

$$
\begin{gathered}
E=\bar{P}\left[\begin{array}{cc}
E_{1} & 0 \\
0 & 0
\end{array}\right] \bar{Q}, \quad A=\bar{P}\left[\begin{array}{ll}
A_{1} & A_{2} \\
A_{3} & A_{4}
\end{array}\right] \bar{Q}, \\
B=\bar{P}\left[\begin{array}{l}
B_{1} \\
B_{2}
\end{array}\right] \widetilde{Q}, \quad C=\widetilde{P}\left[\begin{array}{ll}
C_{1} & C_{2}
\end{array}\right] \bar{Q},
\end{gathered}
$$

where $E_{1} \in \mathbb{R}^{n_{1} \times n_{1}}$ is nonsingular, $A_{4} \in \mathbb{R}^{n_{2} \times n_{2}}$, and $n_{1}+n_{2}=$ $n$, and

$$
\begin{aligned}
& A_{1}=\left[\begin{array}{ll}
A_{11} & A_{12} \\
A_{21} & A_{22}
\end{array}\right], \quad A_{2}=\left[\begin{array}{ll}
A_{13} & A_{14} \\
A_{23} & A_{24}
\end{array}\right], \\
& B_{1}=\left[\begin{array}{cc}
0 & I_{2} \\
0 & 0
\end{array}\right], \quad A_{3}=\left[\begin{array}{ll}
A_{31} & A_{32} \\
A_{41} & A_{42}
\end{array}\right] \text {, } \\
& A_{4}=\left[\begin{array}{ll}
A_{33} & A_{34} \\
A_{43} & A_{44}
\end{array}\right], \quad B_{2}=\left[\begin{array}{cc}
I_{1} & 0 \\
0 & 0
\end{array}\right] \text {, } \\
& C_{1}=\left[\begin{array}{cc}
0 & 0 \\
I_{4} & 0
\end{array}\right], \quad C_{2}=\left[\begin{array}{cc}
I_{3} & 0 \\
0 & 0
\end{array}\right],
\end{aligned}
$$

where $I_{1}, I_{2}, I_{3}$, and $I_{4}$ are $m_{1}$-dimensional, $m_{2}$-dimensional, $l_{1}$-dimensional, and $l_{2}$-dimensional identity matrices, respectively, and $m_{1}+m_{2}=m, l_{1}+l_{2}=l$. Specifically, when $B$ is a column vector, either of $B_{1}$ and $B_{2}$ is in the form $[1,0, \ldots, 0]^{T}$, and the other is in the form $[0,0, \ldots, 0]^{T}$. Likewise, if $C$ is a row vector, the result follows immediately.

Lemma 1 (see [2]). System (4) is impulse free if and only if

$$
\operatorname{rank}\left[\begin{array}{cc}
E & A \\
0 & E
\end{array}\right]=n+\operatorname{rank} E \text {. }
$$

According to Lemma 1, together with (7), system (4) is regular and impulse free if and only if rank $A_{4}=n_{2}$.

Lemma 2 (see [2]). System (6) is impulse free if and only if

$$
\operatorname{rank}\left[\begin{array}{cc}
E+B L C & A \\
0 & E+B L C
\end{array}\right]=n+\operatorname{rank}(E+B L C) .
$$

Lemma 3 (see [2]). For arbitrary matrices $X \in \mathbb{R}^{n \times r}, Y \in$ $\mathbb{R}^{n \times m}$, and $Z \in \mathbb{R}^{l \times r}$, the equality

$$
g_{\dot{U}} r[X+Y U Z]=\min \left\{\operatorname{rank}\left[\begin{array}{ll}
X & Y
\end{array}\right], \operatorname{rank}\left[\begin{array}{c}
X \\
Z
\end{array}\right]\right\}
$$

holds, where $g_{\dot{U}} r[*]$ is the maximal rank of matrix pencil [*] with parameter matrix $U \in \mathbb{R}^{m \times l}$.

\section{Minimum Rank}

In this section, we will give the minimal rank of matrix pencils. In order to obtain this result, we first need to derive the subsequent theorem.
Theorem 4. For arbitrary matrices $G \in \mathbb{R}^{n \times r}, H \in \mathbb{R}^{n \times l}$, and $S \in \mathbb{R}^{m \times r}$, there exists matrix $T \in \mathbb{R}^{m \times l}$ such that

$$
\min _{T} \operatorname{rank}\left[\begin{array}{cc}
G & H \\
S & T
\end{array}\right]=\operatorname{rank}\left[\begin{array}{ll}
G & H
\end{array}\right]+\operatorname{rank}\left[\begin{array}{c}
G \\
S
\end{array}\right]-\operatorname{rank} G .
$$

Proof. For arbitrary matrix $G$, there exist invertible matrices $P$ and $Q$ such that

$$
P G Q=\left[\begin{array}{ll}
I & 0 \\
0 & 0
\end{array}\right]
$$

$$
\begin{aligned}
\operatorname{rank}\left[\begin{array}{cc}
G & H \\
S & T
\end{array}\right] \\
=\operatorname{rank}\left[\begin{array}{ccc}
I & 0 & H_{1} \\
0 & 0 & H_{2} \\
S_{1} & S_{2} & T
\end{array}\right]=\operatorname{rank}\left[\begin{array}{ccc}
I & 0 & 0 \\
0 & 0 & H_{2} \\
0 & S_{2} & T-S_{1} H_{1}
\end{array}\right] \\
=\operatorname{rank} G+\operatorname{rank}\left[\begin{array}{cc}
0 & H_{2} \\
S_{2} & T-S_{1} H_{1}
\end{array}\right] \frac{\min }{\overline{T=S_{1} H_{1}}} \operatorname{rank} G \\
\\
+\operatorname{rank} H_{2}+\operatorname{rank} S_{2},
\end{aligned}
$$

where $P H=\left[\begin{array}{l}H_{1} \\ H_{2}\end{array}\right], S Q=\left[\begin{array}{ll}S_{1} & S_{2}\end{array}\right]$.

From the above fact, it follows that

$$
\min _{T} \operatorname{rank}\left[\begin{array}{cc}
G & H \\
S & T
\end{array}\right]=\operatorname{rank}\left[\begin{array}{cc}
G & H
\end{array}\right]+\operatorname{rank}\left[\begin{array}{c}
G \\
S
\end{array}\right]-\operatorname{rank} G \text {. }
$$

This completes the proof.

Now, we are in a position to derive representation for the minimal rank of matrix pencil.

Theorem 5. For matrix pencil $(E+B L C)$ with a parameter matrix $L$, the equality

$$
\min _{L} \operatorname{rank}(E+B L C)=\operatorname{rank} E-\operatorname{rank} C_{1} E_{1}^{-1} B_{1}
$$

holds.

Proof. If we choose

$$
L=\widetilde{Q}^{-1}\left[\begin{array}{ll}
L_{11} & L_{12} \\
L_{21} & L_{22}
\end{array}\right] \widetilde{P}^{-1},
$$

where $L_{i j} \in \mathbb{R}^{m_{i} \times l_{j}}(i, j=1,2)$, combined with (7) and (8), then

$$
E+B L C=P\left[\begin{array}{cc}
E_{1}+\left[\begin{array}{cc}
L_{22} & 0 \\
0 & 0
\end{array}\right] & {\left[\begin{array}{cc}
L_{21} & 0 \\
0 & 0
\end{array}\right]} \\
{\left[\begin{array}{cc}
L_{12} & 0 \\
0 & 0
\end{array}\right]} & {\left[\begin{array}{cc}
L_{11} & 0 \\
0 & 0
\end{array}\right]}
\end{array}\right] Q .
$$

To obtain the minimal rank of (17), suppose that $L_{11}=0$, $L_{12}=0, L_{21}=0$, and $E_{1}=\left[\begin{array}{ll}E_{11} & E_{12} \\ E_{21} & E_{22}\end{array}\right]$; then we can have

$$
\min _{L} \operatorname{rank}(E+B L C)=\min _{L_{22}} \operatorname{rank}\left[\begin{array}{cc}
E_{11}+L_{22} & E_{12} \\
E_{21} & E_{22}
\end{array}\right] .
$$


By Theorem 4, it follows that

$$
\begin{aligned}
& \min _{L} \operatorname{rank}(E+B L C) \\
& \quad=\operatorname{rank}\left[\begin{array}{ll}
E_{21} & E_{22}
\end{array}\right]+\operatorname{rank}\left[\begin{array}{c}
E_{12} \\
E_{22}
\end{array}\right]-\operatorname{rank} E_{22} .
\end{aligned}
$$

On one hand, we have

$$
\operatorname{rank}\left[\begin{array}{cc}
E_{1} & B_{1} \\
C_{1} & 0
\end{array}\right]=\operatorname{rank} B_{1}+\operatorname{rank} C_{1}+\operatorname{rank} E_{22} .
$$

On the other hand, since $E_{1}$ is nonsingular, it follows that

$$
\begin{aligned}
\operatorname{rank}\left[\begin{array}{cc}
E_{1} & B_{1} \\
C_{1} & 0
\end{array}\right] & =\operatorname{rank}\left[\begin{array}{cc}
E_{1} & 0 \\
C_{1} & -C_{1} E_{1}^{-1} B_{1}
\end{array}\right] \\
& =\operatorname{rank} E_{1}+\operatorname{rank} C_{1} E_{1}^{-1} B_{1} .
\end{aligned}
$$

Thus, the aforementioned fact means that

$$
\min _{L} \operatorname{rank}(E+B L C)=\operatorname{rank} E-\operatorname{rank} C_{1} E_{1}^{-1} B_{1} .
$$

This completes the proof.

For convenience, the following notation is used: for a given matrix $M \in \mathbb{R}^{s \times t}$, the right zero divisor of $M$ is denoted by $S_{M}$, which satisfies $M S_{M}=0$ with $\operatorname{rank} S_{M}=t-\operatorname{rank} M$. Similarly, the left zero divisor of matrix $M$ is denoted by $T_{M}$, satisfying $T_{M} M=0$ with $\operatorname{rank} T_{M}=s-\operatorname{rank} M$.

\section{Main Results}

In this section, derivative output feedback controllers are designed to make the resultant closed loop system regular and impulse free.

It is pointed out in [25] that derivative feedback will eliminate the impulses of descriptor systems if it can be done by proportional feedback. Therefore, the derivative output feedback

$$
u(t)=-L \dot{y}(t)
$$

is used and then the resultant closed loop system

$$
(E+B L C) \dot{x}(t)=A x(t)
$$

is obtained, where $L \in \mathbb{R}^{m \times l}$ is the feedback gain to be determined.

According to the different scope of dynamical order of the resultant closed loop system (24), we divide our main results into four subsections.

\subsection{Impulse Elimination with Maximal Dynamical Order $\tau_{\max }$}

Theorem 6. If

$$
\begin{gathered}
\operatorname{rank}\left[\begin{array}{cc}
E & B
\end{array}\right]>\operatorname{rank}\left[\begin{array}{ll}
E^{T} & C^{T}
\end{array}\right], \\
\operatorname{rank}\left[\begin{array}{c}
T_{L_{11}} A_{34} \\
A_{44}
\end{array}\right]=n-\operatorname{rank}\left[\begin{array}{c}
E \\
C
\end{array}\right],
\end{gathered}
$$

then there exists a derivative output feedback of the following form:

$$
u=-\widetilde{Q}^{-1}\left[\begin{array}{cc}
L_{11} & 0 \\
0 & 0
\end{array}\right] \widetilde{P}^{-1} \dot{y}
$$

such that the resultant closed loop system (24) is regular and impulse free and achieves its maximal dynamical order $\tau_{\max }=$ $\operatorname{rank}\left[E^{T} C^{T}\right]$, where $L_{11} \in \mathbb{R}^{m_{1} \times l_{1}}$ is any matrix of full column rank.

Proof. It follows from Lemma 2 that the resultant closed loop system (24) is regular and impulse free if and only if there exists matrix $L$ such that

$$
\operatorname{rank}\left[\begin{array}{cc}
E+B L C & A \\
0 & E+B L C
\end{array}\right]=n+\operatorname{rank}(E+B L C) .
$$

Assuming that (25) holds, with the help of (11) in Lemma 3, we can have $\operatorname{rank} I_{1}=m_{1}>\operatorname{rank} I_{3}=l_{1}$ and the maximal dynamical order of the system (24) is $\tau_{\max }=$ $\operatorname{rank}\left[\begin{array}{c}E \\ C\end{array}\right]$.

Substituting $L=\widetilde{Q}^{-1}\left[\begin{array}{cc}L_{11} & 0 \\ 0 & 0\end{array}\right] \widetilde{P}^{-1}$ into the left-hand side of (28), matrix manipulation gives

$$
\begin{aligned}
& \operatorname{rank}\left[\begin{array}{cc}
E+B L C & A \\
0 & E+B L C
\end{array}\right] \\
& =\operatorname{rank}\left[\begin{array}{cccc}
E_{1} & 0 & A_{1} & A_{2} \\
0 & {\left[\begin{array}{cc}
L_{11} & 0 \\
0 & 0
\end{array}\right]} & A_{3} & A_{4} \\
0 & 0 & E_{1} & 0 \\
0 & 0 & 0 & {\left[\begin{array}{cc}
L_{11} & 0 \\
0 & 0
\end{array}\right]}
\end{array}\right] \\
& =\operatorname{rank}\left[\begin{array}{ccccc}
E_{1} & 0 & 0 & 0 \\
0 & {\left[\begin{array}{cc}
L_{11} & 0 \\
0 & 0
\end{array}\right]} & 0 & {\left[\begin{array}{cc}
0 & A_{34} \\
0 & A_{44}
\end{array}\right]} \\
0 & 0 & E_{1} & 0 \\
0 & 0 & 0 & {\left[\begin{array}{cc}
L_{11} & 0 \\
0 & 0
\end{array}\right]}
\end{array}\right] \\
& =2 \operatorname{rank}\left[\begin{array}{l}
E \\
C
\end{array}\right]+\operatorname{rank}\left[\begin{array}{c}
T_{L_{11}} A_{34} \\
A_{44}
\end{array}\right] \text {. }
\end{aligned}
$$

It is concluded from (26) and (28) that the resultant closed loop system (24) is regular and impulse free when (25) holds. The corresponding derivative output feedback is provided by (27). This completes the proof.

Theorem 7. If $\operatorname{rank}\left[\begin{array}{ll}E & B\end{array}\right]>\operatorname{rank}\left[E^{T} C^{T}\right]$ and a matrix $L_{12} \in \mathbb{R}^{m_{1} \times l_{2}}$ satisfies

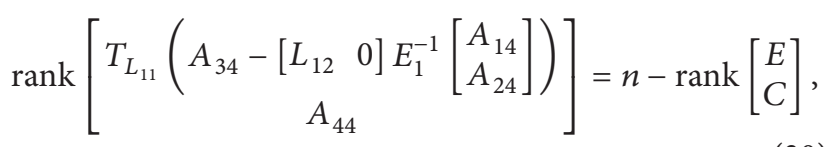

then there exists a derivative output feedback of the following form:

$$
u(t)=-\widetilde{Q}^{-1}\left[\begin{array}{cc}
L_{11} & L_{12} \\
0 & 0
\end{array}\right] \widetilde{P}^{-1} \dot{y}(t)
$$


such that the resultant closed loop system (24) is regular and impulse free and achieves its maximal dynamical order $\tau_{\max }=$ $\operatorname{rank}\left[\begin{array}{ll}E^{T} & C^{T}\end{array}\right]$, where $L_{11}$ is any matrix of full column rank.

Proof. Proceeding as in the proof of Theorem 6, the left-hand side of (28) is equal to

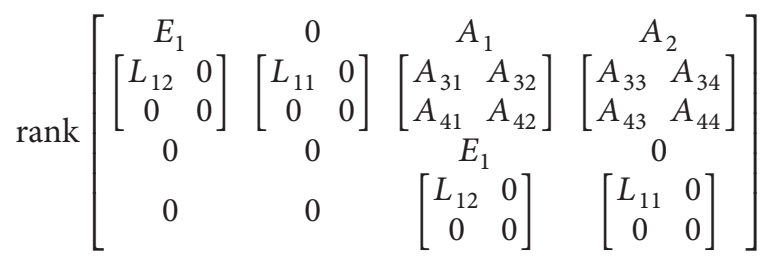

$$
\begin{aligned}
& =\operatorname{rank}\left[\begin{array}{cccc}
E_{1} & 0 & 0 & {\left[\begin{array}{ll}
0 & A_{14} \\
0 & A_{24}
\end{array}\right]} \\
{\left[\begin{array}{cc}
L_{12} & 0 \\
0 & 0
\end{array}\right]} & {\left[\begin{array}{cc}
L_{11} & 0 \\
0 & 0
\end{array}\right]} & 0 & {\left[\begin{array}{ll}
0 & A_{34} \\
0 & A_{44}
\end{array}\right]} \\
0 & 0 & E_{1} & 0 \\
0 & 0 & 0 & {\left[\begin{array}{cc}
L_{11} & 0 \\
0 & 0
\end{array}\right]}
\end{array}\right]
\end{aligned}
$$

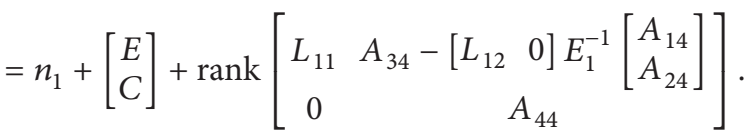

The property of left zero divisor, together with the above discussion, yields that (30) can guarantee regularity and nonimpulsiveness of system (24) in the case that $\operatorname{rank}\left[\begin{array}{ll}E & B\end{array}\right]>$ $\operatorname{rank}\left[\begin{array}{ll}E^{T} & C^{T}\end{array}\right]$. This completes the proof.

Remark 8. Theorems 6 and 7 propose the sufficient conditions under which the resultant closed loop system (24) is regular and impulse free and has the maximal dynamical order in the case of $\operatorname{rank}\left[\begin{array}{ll}E & B\end{array}\right]>\operatorname{rank}\left[\begin{array}{ll}E^{T} & C^{T}\end{array}\right]$. Intuitively, the condition in Theorem 7 , to some extent, relaxes that in Theorem 6 by introducing a parameterized matrix. As proved in the case of $\operatorname{rank}\left[\begin{array}{ll}E & B\end{array}\right]>\operatorname{rank}\left[\begin{array}{ll}E^{T} & C^{T}\end{array}\right]$, the results for the case of $\operatorname{rank}\left[\begin{array}{ll}E & B\end{array}\right] \leq \operatorname{rank}\left[\begin{array}{ll}E^{T} & C^{T}\end{array}\right]$, which integrate the results for the maximal dynamical order case, can also be obtained. For the sake of simplicity, the proof is omitted here.

\section{Theorem 9. If}

$$
\begin{aligned}
& \operatorname{rank}\left[\begin{array}{ll}
E & B
\end{array}\right] \leq \operatorname{rank}\left[\begin{array}{ll}
E^{T} & C^{T}
\end{array}\right], \\
& \operatorname{rank}\left[A_{43} S_{L_{11}} A_{44}\right]=n-\operatorname{rank}\left[\begin{array}{ll}
E & B
\end{array}\right]
\end{aligned}
$$

then there exists a derivative output feedback of the following form:

$$
u=-\widetilde{Q}^{-1}\left[\begin{array}{cc}
L_{11} & 0 \\
0 & 0
\end{array}\right] \widetilde{P}^{-1} \dot{y},
$$

such that the resultant closed loop system (24) is regular and impulse free and achieves its maximal dynamical order $\tau_{\max }=$ $\operatorname{rank}\left[\begin{array}{ll}E & B\end{array}\right]$, where $L_{11}$ is any matrix of full row rank.
Theorem 10. If

$$
\operatorname{rank}\left[\begin{array}{cc}
E & B
\end{array}\right] \leq \operatorname{rank}\left[\begin{array}{ll}
E^{T} & C^{T}
\end{array}\right]
$$

and a matrix $L_{21} \in \mathbb{R}^{m_{2} \times l_{1}}$ satisfies

$$
\begin{aligned}
& \operatorname{rank}\left[\left(A_{43}-\left[\begin{array}{ll}
A_{41} & A_{42}
\end{array}\right] E_{1}^{-1}\left[\begin{array}{c}
L_{21} \\
0
\end{array}\right]\right) S_{L_{11}} A_{44}\right] \\
& =n-\operatorname{rank}\left[\begin{array}{ll}
E & B
\end{array}\right]
\end{aligned}
$$

then there exists a derivative output feedback of the following form:

$$
u(t)=\widetilde{Q}^{-1}\left[\begin{array}{ll}
L_{11} & 0 \\
L_{21} & 0
\end{array}\right] \widetilde{P}^{-1} \dot{y}(t),
$$

such that the resultant closed loop system (24) is regular and impulse free and achieves its maximal dynamical order $\tau_{\max }=$ $\operatorname{rank}\left[\begin{array}{ll}E & B\end{array}\right]$, where $L_{11}$ is any matrix of full row rank.

4.2. Impulse Elimination with Minimal Dynamical Order $\tau_{\min }$. To facilitate the subsequent development and without loss of generality, $E_{1}$ is assumed to be in the following form:

$$
E_{1}=\left[\begin{array}{cc}
E_{11} & {\left[\begin{array}{ll}
E_{12} & 0
\end{array}\right]} \\
{\left[\begin{array}{c}
E_{21} \\
0
\end{array}\right]} & {\left[\begin{array}{ll}
0 & 0 \\
0 & I_{5}
\end{array}\right]}
\end{array}\right]
$$

where the dimension of $E_{11}$ is consistent with that of $L_{22}, E_{12}$ is full column rank, and $E_{21}$ is full row rank.

Remark 11. It should be mentioned that $E_{1}$ can be transformed to form (38) by proper matrix transformations which will not change the structures of $B_{1}$ and $C_{1}$. In order to explain this statement clearly, let $E_{1}$ be partitioned as follows:

$$
E_{1}=\left[\begin{array}{ll}
E_{11} & E_{12} \\
E_{21} & E_{22}
\end{array}\right] .
$$

Since $E_{1}$ is nonsingular, we can have that $\left[\begin{array}{ll}E_{21} & E_{22}\end{array}\right]$ is of full row rank; then there exists a nonsingular matrix $P_{1}$ such that

$$
P_{1}\left[\begin{array}{ll}
E_{21} & E_{22}
\end{array}\right]=\left[\begin{array}{cc}
\widehat{E}_{21} & \widehat{E}_{2} \\
0 & \widehat{E}_{3}
\end{array}\right] .
$$

Thus $\widehat{E}_{3}$ is of full row rank; then there exist nonsingular matrices $P_{2}$ and $Q_{1}$ such that

$$
\begin{gathered}
P_{2} \widehat{E}_{3} Q_{1}=\left[\begin{array}{ll}
0 & I
\end{array}\right], \quad \widehat{E}_{2} Q_{1}=\left[\begin{array}{ll}
\widehat{E}_{22} & \widehat{E}_{23}
\end{array}\right], \\
E_{12} Q_{1}=\left[\begin{array}{ll}
\widehat{E}_{12} & \widehat{E}_{13}
\end{array}\right],
\end{gathered}
$$

which yields that

$$
\left[\begin{array}{ccc}
I & 0 & 0 \\
0 & I & 0 \\
0 & 0 & P_{2}
\end{array}\right]\left[\begin{array}{cc}
I & 0 \\
0 & P_{1}
\end{array}\right] E_{1}\left[\begin{array}{cc}
I & 0 \\
0 & Q_{1}
\end{array}\right]=\left[\begin{array}{ccc}
E_{11} & \widehat{E}_{12} & \widehat{E}_{13} \\
\widehat{E}_{21} & \widehat{E}_{22} & \widehat{E}_{23} \\
0 & 0 & I
\end{array}\right] .
$$


Consequently, some proper matrix transformations show that

$$
E \longrightarrow\left[\begin{array}{ccc}
E_{11} & \widehat{E}_{12} & \widehat{E}_{13} \\
\widehat{E}_{21} & \widehat{E}_{22} & \widehat{E}_{23} \\
0 & 0 & I
\end{array}\right] \longrightarrow\left[\begin{array}{ccc}
E_{11} & \widehat{E}_{12} & 0 \\
\widehat{E}_{21} & \widehat{E}_{22} & 0 \\
0 & 0 & I
\end{array}\right]
$$

The nonsingularity of $E_{1}$ implies that $\left[\begin{array}{ll}\widehat{E}_{21} & \widehat{E}_{22}\end{array}\right]$ is of full row rank and $\left[\begin{array}{l}\widehat{E}_{12} \\ \widehat{E}_{22}\end{array}\right]$ is of full column rank. Repeat the aforementioned step; then the process will terminate if and only if $E_{1}$ can be transformed to

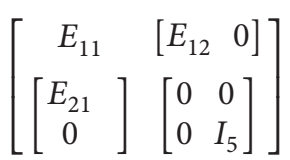

in which $E_{12}$ is full column rank, and $E_{21}$ is full row rank. Otherwise, $E_{1}$ will have the following form:

$$
\left[\begin{array}{cc}
E_{11} & 0 \\
0 & I
\end{array}\right]
$$

Since $E_{11}$ may be rectangular and $E_{1}$ is square, the above form does not always hold. Thus, by proper matrix transformations, $E_{1}$ can be transformed into

$$
\left[\begin{array}{cc}
E_{11} & {\left[\begin{array}{ll}
E_{12} & 0
\end{array}\right]} \\
{\left[\begin{array}{c}
E_{21} \\
0
\end{array}\right]} & {\left[\begin{array}{ll}
0 & 0 \\
0 & I_{5}
\end{array}\right]}
\end{array}\right]
$$

In what follows, it will be found that some results benefit from this special form of $E_{1}$.

Actually, from the proof of Theorem 5, it is shown that the minimal dynamical order of the system (24) is

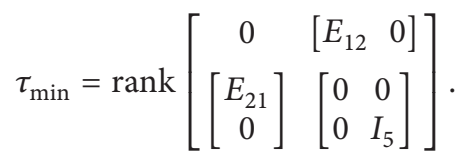

To admit the minimal dynamical order, we present a sufficient condition as follows.

Theorem 12. If the condition

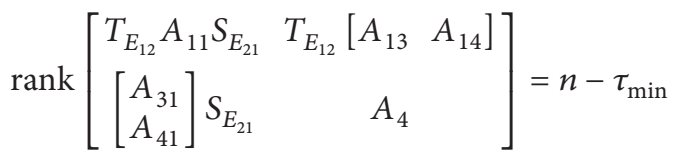

holds and system (4) is impulse free, then there exists a derivative output feedback of the form

$$
u(t)=-\widetilde{Q}^{-1}\left[\begin{array}{cc}
0 & 0 \\
0 & -E_{11}
\end{array}\right] \widetilde{P}^{-1} \dot{y}(t)
$$

such that the resultant closed loop system (24) is regular and impulse free and achieves its minimal dynamical order $\tau_{\min }$.

Proof. Substituting $L=\widetilde{Q}^{-1}\left[\begin{array}{cc}0 & 0 \\ 0 & -E_{11}\end{array}\right] \widetilde{P}^{-1}$ into (28) yields

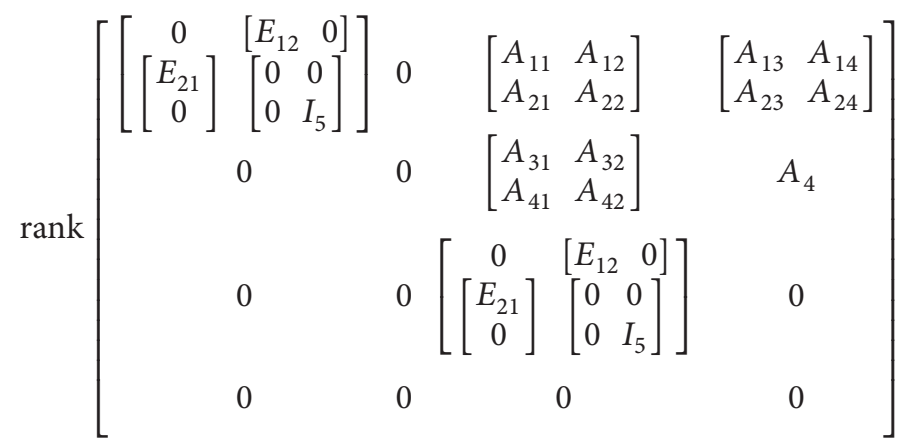

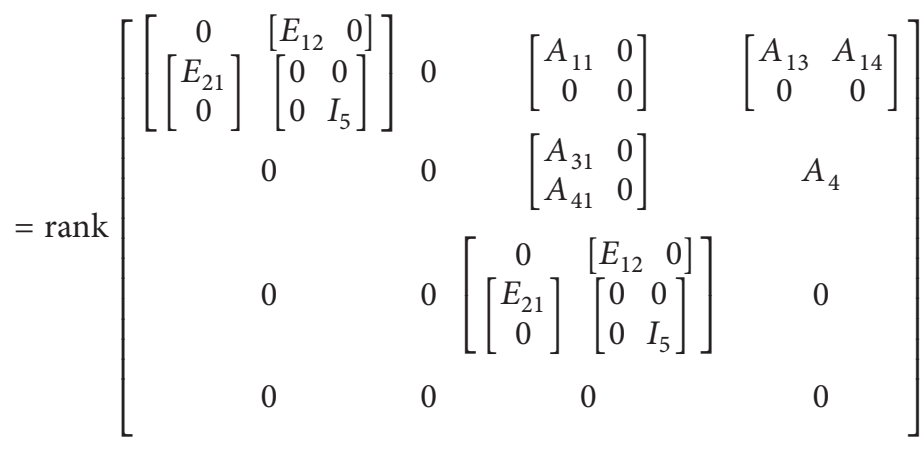

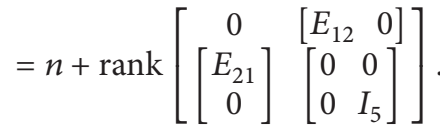


Hence, (28) holds if the condition

$$
\operatorname{rank}\left[\begin{array}{cc}
T_{E_{12}} A_{11} S_{E_{21}} & T_{E_{12}}\left[\begin{array}{ll}
A_{13} & A_{14}
\end{array}\right] \\
{\left[\begin{array}{l}
A_{31} \\
A_{41}
\end{array}\right] S_{E_{21}}} & A_{4}
\end{array}\right]=n-\tau_{\min }
$$

is satisfied. This completes the proof.
In particular, if the $A_{4}$ is nonsingular, condition (48) turns to be

$$
\begin{aligned}
\operatorname{rank} & \left\{T_{E_{12}}\left(A_{11}-\left[\begin{array}{ll}
A_{13} & A_{14}
\end{array}\right] A_{4}^{-1}\left[\begin{array}{l}
A_{31} \\
A_{41}
\end{array}\right]\right) S_{E_{21}}\right\} \\
& =n_{1}-\tau_{\min } .
\end{aligned}
$$

4.3. Impulse Elimination with Dynamical Order in $\left[\tau_{\min }, n_{1}\right]$

Theorem 13. If $L_{\Omega_{1} \cdot \Omega_{2}}$ satisfies

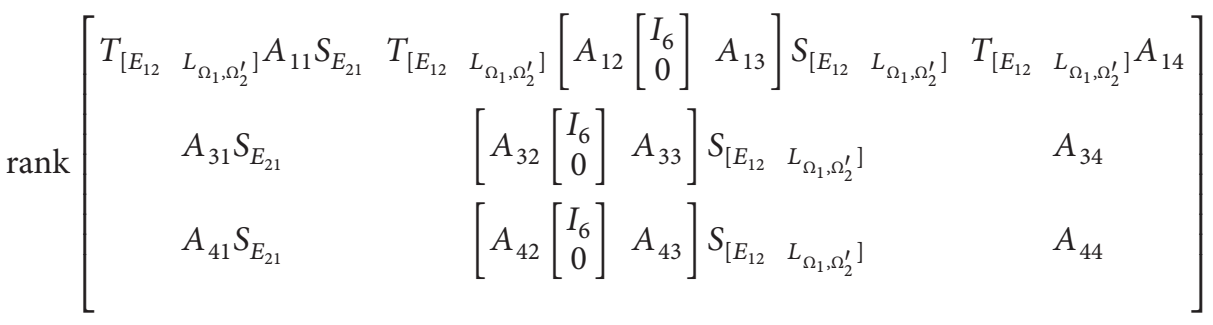

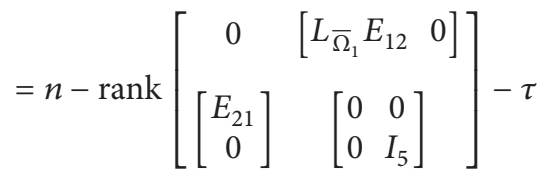

then there exists a derivative output feedback of the form

$$
u=-\widetilde{Q}^{-1}\left[\begin{array}{cc}
0 & 0 \\
L_{\Omega_{1}, \Omega_{2}} & -E_{11}
\end{array}\right] \widetilde{P}^{-1} \dot{y}
$$

such that the resultant closed loop system (24) is regular and impulse free and has the dynamical order

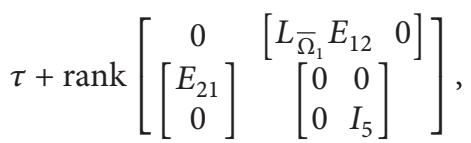

which takes values in the interval $\left[\tau_{\min }, n_{1}\right]$, where $\left[\begin{array}{c}I_{6} \\ 0\end{array}\right]$ is the right zero divisor of $\left[\begin{array}{ll}0 & 0 \\ 0 & I_{5}\end{array}\right]$. Sets $\Omega_{1}, \Omega_{2}^{\prime}$ are, respectively, contained in sets $\left\{1,2, \ldots, m_{2}\right\},\left\{1,2, \ldots, l_{1}\right\} . \bar{\Omega}_{1}, \bar{\Omega}_{2}^{\prime}$ are the complement of sets $\Omega_{1}, \Omega_{2}^{\prime}$ relative to sets $\left\{1,2, \ldots, m_{2}\right\}$, $\left\{1,2, \ldots, l_{1}\right\}$, respectively. $L_{\Omega_{1} \cdot \Omega_{2}^{\prime}}$ is the $m_{2} \times l_{1}$-dimensional matrix whose elements are 1 only in the corresponding rows and columns belonging to the sets $\Omega_{1}, \Omega_{2}$, the remaining elements are 0 , and $L_{\bar{\Omega}_{1}}$ is the $m_{2} \times m_{2}$-dimensional matrix where the main diagonal elements whose rows belong to $\bar{\Omega}_{1}$ are specified to 1 and others are 0 . Obviously, $L_{\bar{\Omega}_{1}}$ is the left zero divisor of $L_{\Omega_{1} \cdot \Omega_{2}^{\prime}}$. Note that $\operatorname{rank} L_{\Omega_{1}, \Omega_{2}^{\prime}}=\tau$.

Proof. Combining (54) into (28) gives

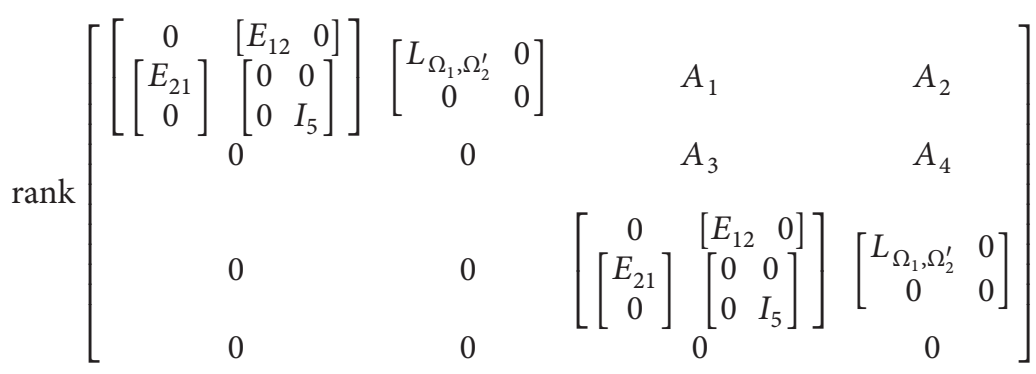




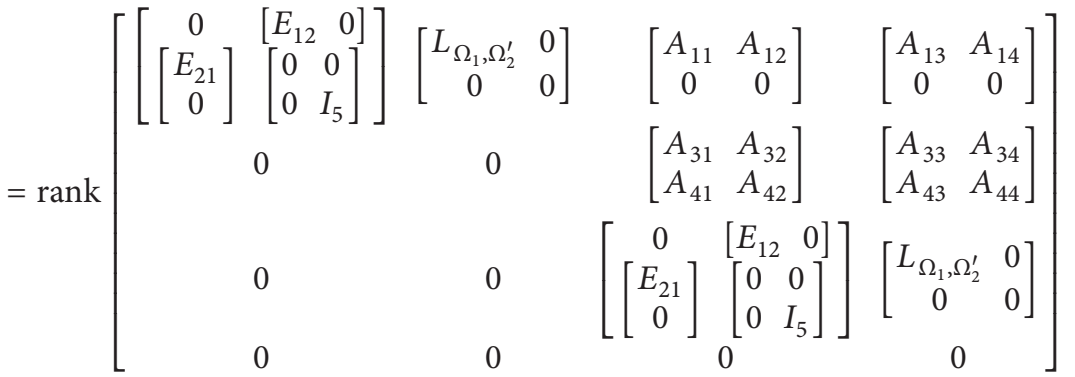

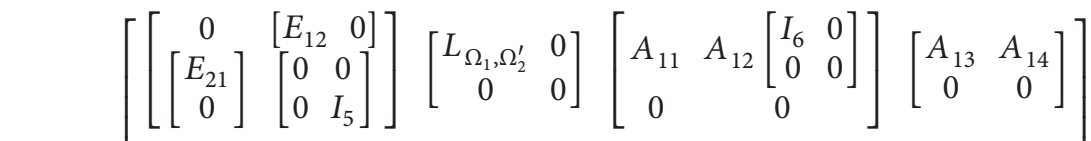

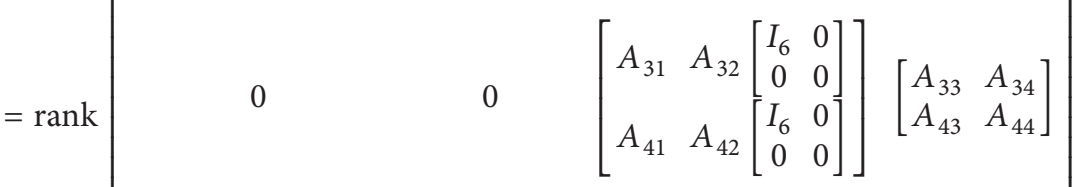

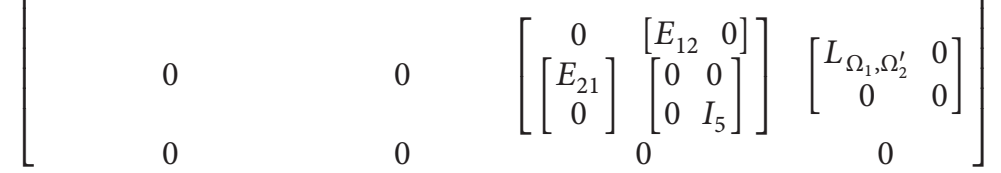

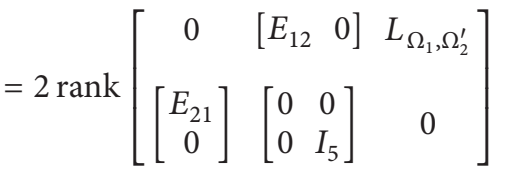

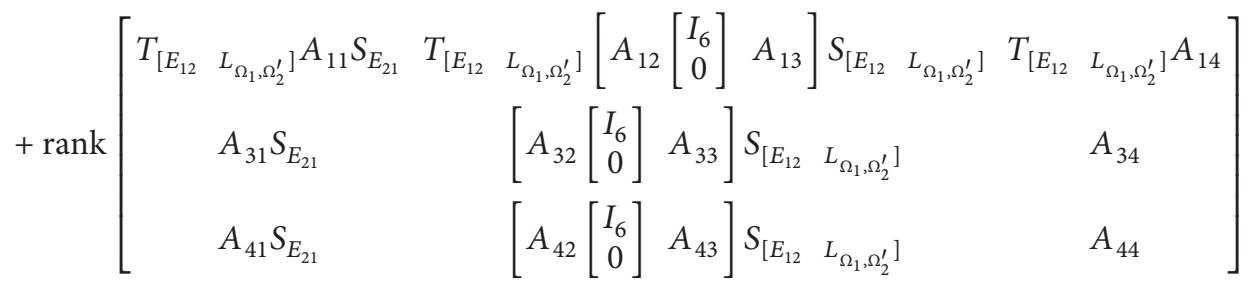

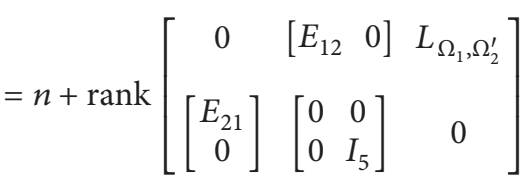

which is equivalent to

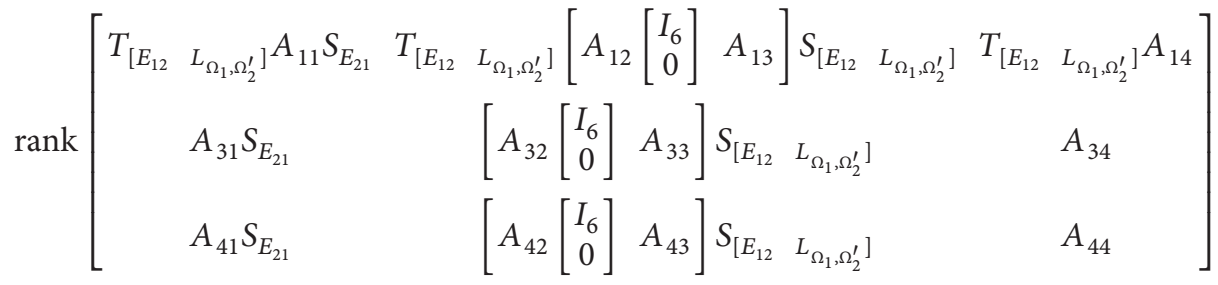

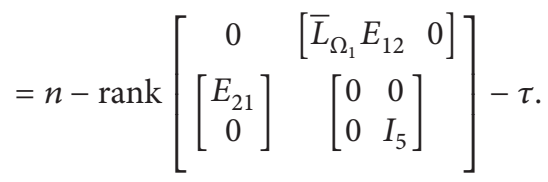

This completes the proof. 


\subsection{Impulse Elimination with Dynamical Order in $\left[n_{1}, \tau_{\max }\right]$}

Theorem 14. If $L_{\Omega_{1}^{\prime}, \Omega_{2}^{\prime}}$ satisfies

$$
\operatorname{rank}\left[\begin{array}{cc}
L_{\bar{\Omega}_{1}^{\prime}} A_{33} L_{\bar{\Omega}_{2}^{\prime}} & L_{\bar{\Omega}_{1}^{\prime}} A_{34} \\
A_{43} L_{\bar{\Omega}_{2}^{\prime}} & A_{44}
\end{array}\right]=n_{2}-\tau
$$

then there exists a derivative output feedback of the form

$$
u=-\widetilde{Q}^{-1}\left[\begin{array}{cc}
L_{\Omega_{1}^{\prime}, \Omega_{2}^{\prime}} & 0 \\
0 & 0
\end{array}\right] \widetilde{P}^{-1} \dot{y}
$$

such that the resultant closed loop system (24) is regular and impulse free and has the dynamical order $n_{1}+\tau$, where sets $\Omega_{1}^{\prime}, \Omega_{2}^{\prime}$ are, respectively, contained in sets $\left\{1,2, \ldots, m_{1}\right\}$, $\left\{1,2, \ldots, l_{1}\right\} . \bar{\Omega}_{1}^{\prime}, \bar{\Omega}_{2}^{\prime}$ are the complement of sets $\Omega_{1}^{\prime}, \Omega_{2}^{\prime}$ relative to sets $\left\{1,2, \ldots, m_{1}\right\}$ and $\left\{1,2, \ldots, l_{1}\right\}$, respectively. $L_{\Omega_{1}^{\prime} \cdot \Omega_{2}^{\prime}}$ is the $m_{1} \times l_{1}$-dimensional matrix whose elements are 1 only in the corresponding rows and columns belonging to the sets $\Omega_{1}^{\prime}$ and $\Omega_{2}^{\prime}$, the remaining elements are 0 , and $L_{\bar{\Omega}_{1}}$ is the $m_{1} \times m_{1}$ dimensional matrix where the main diagonal elements whose rows belong to $\bar{\Omega}_{1}^{\prime}$ are specified to 1 and others are 0 . Obviously, $L_{\bar{\Omega}_{1}^{\prime}}$ is the left zero divisor of $L_{\Omega_{1}^{\prime} \Omega_{2}^{\prime}}$. Similarly, $L_{\bar{\Omega}_{2}^{\prime}}$ is the right zero divisor of $L_{\Omega_{1}^{\prime} \cdot \Omega_{2}^{\prime}}$. Note rank $L_{\Omega_{1}^{\prime} \cdot \Omega_{2}^{\prime}}=\tau$.

Proof. Substitute $L=\widetilde{Q}^{-1}\left[\begin{array}{cc}L_{\Omega_{1}^{\prime}, \Omega_{2}^{\prime}} & 0 \\ 0 & 0\end{array}\right] \widetilde{P}^{-1}$ into (28); then we obtain

$$
\operatorname{rank}\left[\begin{array}{ccccc}
E_{1} & 0 & A_{1} & A_{2} & \\
0 & \left(\begin{array}{cc}
L_{\Omega_{1}^{\prime}, \Omega_{2}^{\prime}} & 0 \\
0 & 0
\end{array}\right) & A_{3} & A_{4} & \\
0 & 0 & E_{1} & 0 & \\
0 & 0 & 0 & \left(\begin{array}{rl}
L_{\Omega_{1}^{\prime}, \Omega_{2}^{\prime}} & 0 \\
0 & 0
\end{array}\right)
\end{array}\right]=n+n_{1}+\tau
$$

which is equivalent to

$$
\operatorname{rank}\left[\begin{array}{cc}
L_{\bar{\Omega}_{1}^{\prime}} A_{33} L_{\bar{\Omega}_{2}^{\prime}} & L_{\bar{\Omega}_{1}^{\prime}} A_{34} \\
A_{43} L_{\bar{\Omega}_{2}^{\prime}} & A_{44}
\end{array}\right]=n_{2}-\tau .
$$

This completes the proof.

\section{Illustrative Examples}

This section presents three numerical examples to verify the effectiveness of the results obtained in this paper.

Example 1. Consider the circuit depicted in Figure 1, which is taken form [26]. As shown, besides an independent voltage source, a capacitor, and an inductor, the circuit also includes a current-controlled current source.

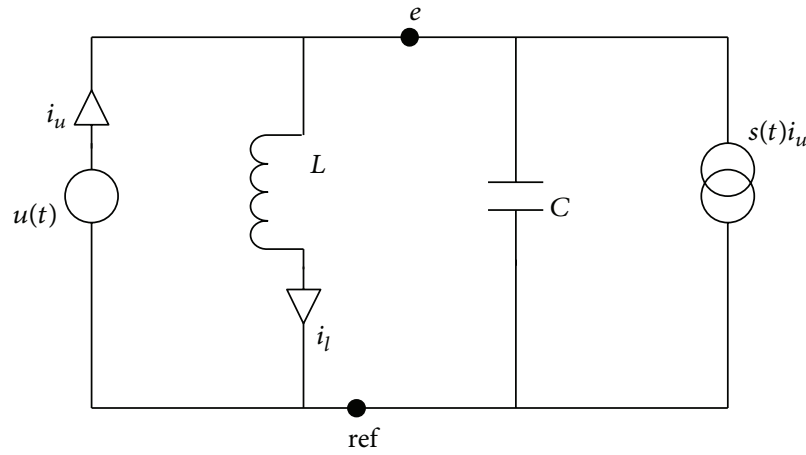

FIGURE 1: A circuit system.

By referring to [26], we can formulate the system model of the circuit system as follows:

$$
\begin{gathered}
(C e)^{\prime}+i_{l}+(s(t)-1) i_{u}=0, \\
\left(L i_{l}\right)^{\prime}-e=0, \\
e=u(t) .
\end{gathered}
$$

It should be noticed that when the input of the system is the step function, pulse current phenomenon will appear and the circuit may be damaged. Thus, eliminating the impulse of the system is necessary. By normalizing $C=L=1$ and letting $a=s(t)-1 \neq 0$, provided that we define $x^{T}=\left[\begin{array}{lll}e^{T} & i_{l}^{T} & i_{u}^{T}\end{array}\right]$ and $y=i_{u}$, then the system (62) can be written in the descriptor form

$$
E \dot{x}=A x+B u, \quad y=C x
$$

with

$$
\begin{array}{cc}
E=\left[\begin{array}{lll}
1 & 0 & 0 \\
0 & 1 & 0 \\
0 & 0 & 0
\end{array}\right], & A=\left[\begin{array}{ccc}
0 & 1 & a \\
1 & 0 & 0 \\
-1 & 0 & 0
\end{array}\right], \\
B=\left[\begin{array}{l}
0 \\
0 \\
1
\end{array}\right], & C=\left[\begin{array}{lll}
0 & 0 & 1
\end{array}\right] .
\end{array}
$$
facts:

Indeed, some matrix computations give the following

(1)

$$
\operatorname{det}(E-A)=\operatorname{det}\left[\begin{array}{ccc}
1 & -1 & a \\
1 & 1 & 0 \\
-1 & 0 & 0
\end{array}\right] \neq 0,
$$

means that system (63) is regular;

(2)

$$
\begin{aligned}
\operatorname{rank}\left[\begin{array}{cc}
E & A \\
0 & E
\end{array}\right] & =\operatorname{rank}\left[\begin{array}{cccccc}
1 & 0 & 0 & 0 & 1 & a \\
0 & 1 & 0 & -1 & 0 & 0 \\
0 & 0 & 0 & 1 & 0 & 0 \\
0 & 0 & 0 & 1 & 0 & 0 \\
0 & 0 & 0 & 0 & 1 & 0 \\
0 & 0 & 0 & 0 & 0 & 0
\end{array}\right] \\
& =4<5=n+\operatorname{rank} E,
\end{aligned}
$$


(3)

$$
\operatorname{rank} E=\operatorname{rank}\left[\begin{array}{ccc}
1 & 0 & 0 \\
0 & 1 & 0 \\
0 & 0 & 0
\end{array}\right]=2,
$$

thus the dynamic order is 2 .

It is easy to verify that the coefficient matrices of (63) fall into the case of Theorem 9, and it follows that the derivative output feedback of the form $u=-L \dot{y}$ with a nonzero scalar $L$ can eliminate the impulsive behavior and furthermore make the resultant closed loop system possess maximal dynamical order. After applying the above feedback controller, the resultant closed loop system is

$$
\left[\begin{array}{lll}
1 & 0 & 0 \\
0 & 1 & 0 \\
0 & 0 & L
\end{array}\right] \dot{x}=A x .
$$

From Lemma 2, it is shown that

$$
\begin{aligned}
\operatorname{rank} & \begin{array}{c}
E+B L C \\
0
\end{array} \quad A \\
& =\operatorname{rank}\left[\begin{array}{cccccc}
1 & 0 & 0 & 0 & 1 & a \\
0 & 1 & 0 & 1 & 0 & 0 \\
0 & 0 & L & -1 & 0 & 0 \\
0 & 0 & 0 & 1 & 0 & 0 \\
0 & 0 & 0 & 0 & 1 & 0 \\
0 & 0 & 0 & 0 & 0 & L
\end{array}\right]=6 \\
& =n+\operatorname{rank}(E+B L C)
\end{aligned}
$$

which means that the resultant closed loop system (68) is impulse free.

Actually, for the resultant closed loop system (68), if scalar $L=0$, then the resultant closed loop system (68) possesses dynamical order 2 (the minimal dynamical order by referring to Lemma 3). It is easy to find that the resultant closed loop systems by any derivative output feedback whose dynamical order is 2 are impulsive.

In order to compare our results with [24], the parameters that arise in Corollary 3 of [24] are shown as

$$
\begin{gathered}
r_{e \gamma}=3, \quad r_{e b}=3, \quad r_{e b \gamma}=3, \quad T_{e b}=\left[\begin{array}{l}
0 \\
0 \\
0
\end{array}\right], \\
S_{e \gamma}=\left[\begin{array}{l}
0 \\
0 \\
0
\end{array}\right], \quad \widetilde{T}_{e \gamma}=\left[\begin{array}{l}
0 \\
0 \\
1
\end{array}\right], \quad \widetilde{S}_{e b}=\left[\begin{array}{l}
0 \\
0 \\
1
\end{array}\right],
\end{gathered}
$$

where $\Gamma=C$ with $C$ defined in this paper.

Combining (63) with (70), we can have

$$
\begin{gathered}
\operatorname{rank}\left(\widetilde{T}_{e \gamma}^{T} A \widetilde{S}_{e b}\right)=0, \quad \operatorname{rank}\left(\widetilde{T}_{e \gamma}^{T} A S_{e \gamma}\right)=0, \\
\operatorname{rank}\left(T_{e b}^{T} A \widetilde{S}_{e b}^{T}\right)=0, \quad \operatorname{rank}\left(T_{e b}^{T} A S_{e \gamma}\right)=0, \\
\operatorname{rank}\left(\left(\widetilde{T}_{e \gamma}^{0}\right)^{T}\left[\begin{array}{l}
A \\
C
\end{array}\right] S_{e \gamma}\right)=0 .
\end{gathered}
$$

After a little manipulation, it is easily checked that the parameters in this case satisfy the conditions of Corollary 3 in [24], from which only the dynamical order 3 can be achieved such that resultant closed loop system is both regular and impulse free by derivative output feedback. Although the criteria in this paper are only sufficient, from this example, it can be observed that our results are not more conservative to use the derivative output feedback to eliminate impulse than Corollary 3 in [24]. Compared with [24], the design process for derivative output feedback is not guaranteed in a numerically stable way, but this method may provide a simple way to realize regularity and nonimpulsiveness under certain circumstance.

Example 2. Consider a descriptor system

$$
E \dot{x}=A x+B u, \quad y=C x
$$

with the following parameters:

$$
\begin{array}{cc}
E=\left[\begin{array}{llll}
1 & 0 & 0 & 0 \\
0 & 0 & 0 & 1 \\
0 & 0 & 0 & 0 \\
0 & 0 & 0 & 0
\end{array}\right], \quad A=\left[\begin{array}{cccc}
-1 & 2 & 3 & 4 \\
3 & 1 & 4 & -1.5 \\
1.5 & -1 & 1 & -2 \\
-2.5 & 0 & 0 & -1
\end{array}\right], \\
B=\left[\begin{array}{cc}
1 & -2 \\
-0.5 & 1 \\
-3 & 2 \\
0 & 0
\end{array}\right], \quad C=\left[\begin{array}{llll}
0 & -1 & 0 & 1
\end{array}\right] .
\end{array}
$$

Simple manipulations show that

(1)

$$
\operatorname{det}(E-A)=\left[\begin{array}{cccc}
2 & -2 & -3 & -4 \\
-3 & -1 & -4 & 2.5 \\
-1.5 & 1 & -1 & 2 \\
2.5 & 0 & 0 & 1
\end{array}\right] \neq 0
$$

implies that system (72) is regular;

(2)

$$
\operatorname{rank}\left[\begin{array}{cc}
E & A \\
0 & E
\end{array}\right]=\operatorname{rank}\left[\begin{array}{cccccccc}
1 & 0 & 0 & 0 & -1 & 2 & 3 & 4 \\
0 & 0 & 0 & 1 & 3 & 1 & 4 & -1.5 \\
0 & 0 & 0 & 0 & 1.5 & -1 & 1 & -2 \\
0 & 0 & 0 & 0 & -2.5 & 0 & 0 & -1 \\
0 & 0 & 0 & 0 & 1 & 0 & 0 & 0 \\
0 & 0 & 0 & 0 & 0 & 0 & 0 & 1 \\
0 & 0 & 0 & 0 & 0 & 0 & 0 & 0 \\
0 & 0 & 0 & 0 & 0 & 0 & 0 & 0
\end{array}\right]
$$$$
=5<6=n+\operatorname{rank} E \text {, }
$$

(3) the dynamic order is 2 ; 
(4)

$$
\begin{aligned}
& \operatorname{rank}\left[\begin{array}{ccc}
E & A & B \\
0 & E & 0
\end{array}\right] \\
& =\operatorname{rank}\left[\begin{array}{cccccccccc}
1 & 0 & 0 & 0 & -1 & 2 & 3 & 4 & 1 & -2 \\
0 & 0 & 0 & 1 & 3 & 1 & 4 & -1.5 & -0.5 & 1 \\
0 & 0 & 0 & 0 & 1.5 & -1 & 1 & -3 & 2 & 0 \\
0 & 0 & 0 & 0 & -2.5 & 0 & 0 & -1 & 0 & 0 \\
0 & 0 & 0 & 0 & 1 & 0 & 0 & 0 & 0 & 0 \\
0 & 0 & 0 & 0 & 0 & 0 & 0 & 1 & 0 & 0 \\
0 & 0 & 0 & 0 & 0 & 0 & 0 & 0 & 0 & 0 \\
0 & 0 & 0 & 0 & 0 & 0 & 0 & 0 & 0 & 0
\end{array}\right]
\end{aligned}
$$

$=5<6=n+\operatorname{rank} E$,

yields that the system (72) is not impulse controllable. Consequently, we cannot use the state feedback to eliminate the impulse.

Selecting

$$
\begin{gathered}
\bar{P}=\left[\begin{array}{cccc}
1 & 0 & 0 & 0 \\
0.5 & 1 & 0 & 0 \\
0 & 0 & 1 & 0 \\
0 & 0 & 0 & 1
\end{array}\right], \quad \bar{Q}=\left[\begin{array}{cccc}
0 & 1 & 0 & 0 \\
1 & 0 & -1 & 0 \\
0 & 0 & 0 & 1 \\
1 & 0 & 0 & 0
\end{array}\right] \widetilde{P}=1, \\
\widetilde{Q}=\left[\begin{array}{cc}
-3 & 2 \\
1 & -2
\end{array}\right],
\end{gathered}
$$

together with (7) and (8), then we have

$$
\begin{aligned}
& \bar{P} E \bar{Q}=\left[\begin{array}{llll}
1 & 0 & 0 & 0 \\
0 & 1 & 0 & 0 \\
0 & 0 & 0 & 0 \\
0 & 0 & 0 & 0
\end{array}\right], \quad \bar{P} A \bar{Q}=\left[\begin{array}{cccc}
-4 & 6 & -2 & 3 \\
1.25 & 2.5 & -2 & 5.5 \\
3 & -3 & 1 & 1 \\
-2 & -1 & 0 & 0
\end{array}\right] \text {, } \\
& \bar{P} B \widetilde{Q}=\left[\begin{array}{ll}
0 & 1 \\
0 & 0 \\
1 & 0 \\
0 & 0
\end{array}\right], \quad \widetilde{P} C \bar{Q}=\left[\begin{array}{llll}
0 & 0 & 1 & 0
\end{array}\right] .
\end{aligned}
$$

Using Lemma 3 and Theorem 5, we obtain that the range of possibly dynamical orders of the resultant closed loop system assigned by derivative output feedback is $\{2,3\}$.

From (78), it is shown that

$$
A_{33}=1, \quad A_{34}=1, \quad A_{43}=0, \quad A_{44}=0
$$

choose $L_{\Omega_{1}, \Omega_{2}^{\prime}}=1$ which implies $L_{\bar{\Omega}_{1}}=L_{\bar{\Omega}_{2}^{\prime}}=0$; by applying Theorem 13, we can obtain that

$$
\begin{aligned}
\operatorname{rank}\left[\begin{array}{cc}
A_{33} L \bar{\Omega}_{2}^{\prime} & A_{34} \\
A_{43} L \bar{\Omega}_{2}^{\prime} & A_{44}
\end{array}\right] & =\operatorname{rank}\left[\begin{array}{ll}
0 & 1 \\
0 & 0
\end{array}\right] \\
& =1=4-\operatorname{rank}\left[\begin{array}{ll}
1 & 0 \\
0 & 1
\end{array}\right]-1
\end{aligned}
$$

which satisfies condition (53); thus there exists a derivative output feedback

$$
u=\left[\begin{array}{c}
5 \\
7.5
\end{array}\right] \dot{y}
$$

such that the resultant closed loop system is impulse free and achieves its minimal dynamical order 2 at the same time.

Unfortunately, by checking the conditions in the maximal dynamical order section, we cannot determine whether the impulse of the resultant closed loop system can be eliminated by derivative output feedback in the case of maximal dynamical order. Therefore, substituting the derivative output feedback of the generic form

$$
u=-\widetilde{Q}^{-1}\left[\begin{array}{l}
L_{1} \\
L_{2}
\end{array}\right] \widetilde{P}^{-1} \dot{y}
$$

with $L_{1} \neq 0$ into the system (72) results in the following resultant closed loop system:

$$
\bar{P}\left[\begin{array}{cccc}
1 & 0 & L_{2} & 0 \\
0 & 1 & 0 & 0 \\
0 & 0 & L_{1} & 0 \\
0 & 0 & 0 & 0
\end{array}\right] \bar{Q} \dot{x}=A x .
$$

According to

$$
\operatorname{rank}\left[\begin{array}{cc}
E+B L C & A \\
0 & E+B L C
\end{array}\right]
$$

$$
=\operatorname{rank}\left[\begin{array}{cccccccc}
1 & 0 & L_{2} & 0 & -4 & 6 & -2 & -3 \\
0 & 1 & 0 & 0 & 1.25 & 2.5 & -2 & 5.5 \\
0 & 0 & L_{1} & 0 & 3 & -3 & 1 & 1 \\
0 & 0 & 0 & 0 & -2 & -1 & 0 & 0 \\
0 & 0 & 0 & 0 & 1 & 0 & L_{2} & 0 \\
0 & 0 & 0 & 0 & 0 & 1 & 0 & 0 \\
0 & 0 & 0 & 0 & 0 & 0 & L_{1} & 0 \\
0 & 0 & 0 & 0 & 0 & 0 & 0 & 0
\end{array}\right]
$$

$$
=6<4+3=n+\operatorname{rank}(E+B L C)
$$

it is shown that the resultant closed loop system is not impulse free. Therefore, the resultant closed loop system (83) cannot achieve the maximal dynamical order and be impulse free by any derivative output feedback simultaneously.

We are now turning to the comparison between our results and [24]. An easy calculation gives the parameters in [24]:

$$
\begin{gathered}
r_{e \gamma}=3, \quad r_{e b}=3, \quad r_{e b \gamma}=4, \\
T_{e b}=\left[\begin{array}{l}
0 \\
0 \\
0 \\
1
\end{array}\right], \quad S_{e \gamma}=\left[\begin{array}{l}
0 \\
0 \\
1 \\
0
\end{array}\right], \quad \widetilde{T}_{e \gamma}=\left[\begin{array}{ll}
0 & 0 \\
0 & 0 \\
1 & 0 \\
0 & 1
\end{array}\right], \\
\widetilde{S}_{e b}=\left[\begin{array}{ccc}
0 & 0 & 0 \\
1 & -1 & 0 \\
0 & 0 & 1 \\
1 & 0 & 0
\end{array}\right]
\end{gathered}
$$


from which we have

$$
\begin{gathered}
\operatorname{rank}\left(\widetilde{T}_{e \gamma}^{T} A \widetilde{S}_{e b}\right)=\operatorname{rank}\left[\begin{array}{ccc}
3 & 1 & 1 \\
-2 & 0 & 0
\end{array}\right]=2, \\
\operatorname{rank}\left(\widetilde{T}_{e \gamma}^{T} A S_{e \gamma}\right)=\operatorname{rank}\left[\begin{array}{l}
1 \\
0
\end{array}\right]=1, \\
\operatorname{rank}\left(T_{e b}^{T} A \widetilde{S}_{e b}\right)=\operatorname{rank}\left[\begin{array}{ccc}
-2 & 0 & 0
\end{array}\right]=1, \\
\operatorname{rank}\left(T_{e b}^{T} A S_{e \gamma}\right)=0
\end{gathered}
$$

By checking the conditions of Corollary 3 in [24], we obtain that the dynamical order 2 can be assigned by derivative output feedback, with which the resultant closed loop system is regular and impulse free. Thus, this example implies that both our results and Corollary 3 in [24] have the same ability to deal with the impulse elimination problems by derivative output feedback.

Example 3. Consider a descriptor system

$$
E \dot{x}=A x+B u, \quad y=C x
$$

with the following parameters:

$$
\begin{gathered}
E=\left[\begin{array}{lllll}
1 & 0 & 0 & 0 & 0 \\
0 & 1 & 0 & 0 & 0 \\
0 & 0 & 0 & 0 & 0 \\
0 & 0 & 0 & 0 & 0 \\
0 & 0 & 0 & 0 & 0
\end{array}\right], \quad A=\left[\begin{array}{ccccc}
-1 & 1 & 0 & -2 & 0 \\
1 & 0 & 0 & 0 & 1 \\
1 & 1 & 0 & 0 & 0 \\
1 & 0 & 0 & 0 & 1 \\
2 & 0 & 0 & 1 & 0
\end{array}\right], \\
B^{T}=C=\left[\begin{array}{lllll}
0 & 1 & 0 & 0 & 0 \\
0 & 0 & 1 & 0 & 0 \\
0 & 0 & 0 & 1 & 0
\end{array}\right] .
\end{gathered}
$$

It is easy to show that

(1)

$$
\operatorname{det}(E-A)=\left[\begin{array}{ccccc}
2 & -1 & 0 & 2 & 0 \\
-1 & 1 & 0 & 0 & -1 \\
-1 & -1 & 0 & 0 & 0 \\
-1 & 0 & 0 & 0 & -1 \\
-2 & 0 & 0 & -1 & 0
\end{array}\right] \neq 0
$$

means that the system (87) is regular;

(2)

$$
\begin{aligned}
& \operatorname{rank}\left[\begin{array}{cc}
E & A \\
0 & E
\end{array}\right] \\
& =\operatorname{rank}\left[\begin{array}{cccccccccc}
1 & 0 & 0 & 0 & 0 & -1 & 1 & 0 & -2 & 0 \\
0 & 1 & 0 & 0 & 0 & 1 & 0 & 0 & 0 & 1 \\
0 & 0 & 0 & 0 & 0 & 1 & 1 & 0 & 0 & 0 \\
0 & 0 & 0 & 0 & 0 & 1 & 0 & 0 & 0 & 1 \\
0 & 0 & 0 & 0 & 0 & 2 & 0 & 0 & 1 & 0 \\
0 & 0 & 0 & 0 & 0 & 1 & 0 & 0 & 0 & 0 \\
0 & 0 & 0 & 0 & 0 & 0 & 1 & 0 & 0 & 0 \\
0 & 0 & 0 & 0 & 0 & 0 & 0 & 0 & 0 & 0 \\
0 & 0 & 0 & 0 & 0 & 0 & 0 & 0 & 0 & 0 \\
0 & 0 & 0 & 0 & 0 & 0 & 0 & 0 & 0 & 0
\end{array}\right] \\
& =6<7=n+\text { rank } E
\end{aligned}
$$

thus the system (87) is impulsive;
(3) the dynamical order is 2 .

If we choose the following transformation matrices associated with (7) and (8):

$$
\begin{gathered}
\bar{P}=\bar{Q}=\left[\begin{array}{lllll}
0 & 1 & 0 & 0 & 0 \\
1 & 0 & 0 & 0 & 0 \\
0 & 0 & 1 & 0 & 0 \\
0 & 0 & 0 & 1 & 0 \\
0 & 0 & 0 & 0 & 1
\end{array}\right], \quad \widetilde{P}=\left[\begin{array}{lll}
0 & 0 & 1 \\
1 & 0 & 0 \\
0 & 1 & 0
\end{array}\right], \\
\widetilde{Q}=\left[\begin{array}{lll}
0 & 1 & 0 \\
0 & 0 & 1 \\
1 & 0 & 0
\end{array}\right],
\end{gathered}
$$

then we have

$$
\begin{array}{cc}
\bar{P} E \bar{Q}=\left[\begin{array}{lllll}
1 & 0 & 0 & 0 & 0 \\
0 & 1 & 0 & 0 & 0 \\
0 & 0 & 0 & 0 & 0 \\
0 & 0 & 0 & 0 & 0 \\
0 & 0 & 0 & 0 & 0
\end{array}\right], & \bar{P} A \bar{Q}=\left[\begin{array}{ccccc}
0 & 1 & 0 & 0 & 1 \\
1 & -1 & 0 & -2 & 0 \\
1 & 1 & 0 & 0 & 0 \\
0 & 1 & 0 & 0 & 1 \\
0 & 2 & 0 & 1 & 0
\end{array}\right], \\
\bar{P} B \widetilde{Q}=\left[\begin{array}{lll}
0 & 0 & 1 \\
0 & 0 & 0 \\
1 & 0 & 0 \\
0 & 1 & 0 \\
0 & 0 & 0
\end{array}\right], & \widetilde{P} C \bar{Q}=\left[\begin{array}{lllll}
0 & 0 & 1 & 0 & 0 \\
0 & 0 & 0 & 1 & 0 \\
1 & 0 & 0 & 0 & 0
\end{array}\right] .
\end{array}
$$

Combining the above matrix with Lemma 3 and Theorem 5 , we obtain that the range of possibly dynamical orders of the resultant closed loop system assigned by derivative output feedback is $\{1,2,3,4\}$.

Provided that dynamical order of the resultant closed loop system is 1 or 4 , corresponding to minimal dynamical order or maximal dynamical order, respectively, by checking the associated conditions proposed in this paper, we cannot find a derivative output feedback to meet the need for nonimpulsiveness in the case of minimal dynamical order or maximal dynamical order. Actually, for this example, any derivative output feedback cannot realize the goal both for desirable dynamical order (minimal case or maximal case) and for nonimpulsiveness, which is illustrated by the detailed analysis below.

Without loss of generality, it is assumed that

$$
u=-L \dot{y}=\left[\begin{array}{lll}
L_{11} & L_{12} & L_{13} \\
L_{21} & L_{22} & L_{23} \\
L_{31} & L_{32} & L_{33}
\end{array}\right] \dot{y}
$$

which gives the resultant closed loop system

$$
\left[\begin{array}{ccccc}
1 & 0 & 0 & 0 & 0 \\
0 & 1+L_{11} & L_{12} & L_{13} & 0 \\
0 & L_{21} & L_{22} & L_{23} & 0 \\
0 & L_{31} & L_{32} & L_{33} & 0 \\
0 & 0 & 0 & 0 & 0
\end{array}\right] \dot{x}=A x .
$$


(1) When $L=\left[\begin{array}{ccc}-1 & 0 & 0 \\ 0 & 0 & 0 \\ 0 & 0 & 0\end{array}\right]$, the dynamical order of the resultant closed loop system (94) is 1 , and

$$
\begin{gathered}
\operatorname{rank}\left[\begin{array}{cccc}
E+B L C & A \\
0 & E+B L C
\end{array}\right] \\
=\operatorname{rank}\left[\begin{array}{cccccccccc}
1 & 0 & 0 & 0 & 0 & -1 & 1 & 0 & -2 & 0 \\
0 & 0 & 0 & 0 & 0 & 1 & 0 & 0 & 0 & 1 \\
0 & 0 & 0 & 0 & 0 & 1 & 1 & 0 & 0 & 0 \\
0 & 0 & 0 & 0 & 0 & 1 & 0 & 0 & 0 & 1 \\
0 & 0 & 0 & 0 & 0 & 2 & 0 & 0 & 1 & 0 \\
0 & 0 & 0 & 0 & 0 & 0 & 1 & 0 & 0 & 0 \\
0 & 0 & 0 & 0 & 0 & 0 & 0 & 0 & 0 & 0 \\
0 & 0 & 0 & 0 & 0 & 0 & 0 & 0 & 0 & 0 \\
0 & 0 & 0 & 0 & 0 & 0 & 0 & 0 & 0 & 0 \\
0 & 0 & 0 & 0 & 0 & 0 & 0 & 0 & 0 & 0
\end{array}\right] \\
=5<5+1=n+\operatorname{rank}(E+B L C)
\end{gathered}
$$

means that the resultant closed loop system is not impulse free. Therefore, the resultant closed loop system (94) cannot achieve the minimal dynamical order and be impulse free simultaneously.

(2) When $L=\left[\begin{array}{lll}1 & 0 & 0 \\ 0 & 1 & 0 \\ 0 & 0 & 1\end{array}\right]$, the dynamical order of the resultant closed loop system (94) is 4 , whereas the resultant closed loop system is not impulse free which can be explained by the following fact:

$$
\begin{aligned}
\operatorname{rank}\left[\begin{array}{cccc}
E+B L C & A \\
0 & E+B L C
\end{array}\right] \\
=\operatorname{rank}\left[\begin{array}{cccccccccc}
1 & 0 & 0 & 0 & 0 & -1 & 1 & 0 & -2 & 0 \\
0 & 1 & 0 & 0 & 0 & 1 & 0 & 0 & 0 & 1 \\
0 & 0 & 1 & 0 & 0 & 1 & 1 & 0 & 0 & 0 \\
0 & 0 & 0 & 1 & 0 & 1 & 0 & 0 & 0 & 1 \\
0 & 0 & 0 & 0 & 0 & 2 & 0 & 0 & 1 & 0 \\
0 & 0 & 0 & 0 & 0 & 1 & 0 & 0 & 0 & 0 \\
0 & 0 & 0 & 0 & 0 & 0 & 1 & 0 & 0 & 0 \\
0 & 0 & 0 & 0 & 0 & 0 & 0 & 1 & 0 & 0 \\
0 & 0 & 0 & 0 & 0 & 0 & 0 & 0 & 1 & 0 \\
0 & 0 & 0 & 0 & 0 & 0 & 0 & 0 & 0 & 0
\end{array}\right] \\
=8<5+4=n+\operatorname{rank}(E+B L C) .
\end{aligned}
$$

Therefore, the resultant closed loop system (94) cannot achieve the maximal dynamical order and be impulse free at the same time.

It is observed from (92) that

$$
\begin{gathered}
A_{11}=0, \quad A_{13}=\left[\begin{array}{ll}
0 & 0
\end{array}\right] \\
A_{14}=1, \quad A_{31}=\left[\begin{array}{l}
1 \\
0
\end{array}\right], \quad A_{41}=0 \\
A_{33}=\left[\begin{array}{ll}
0 & 0 \\
0 & 0
\end{array}\right], \quad A_{34}=\left[\begin{array}{l}
0 \\
1
\end{array}\right], \quad A_{43}=\left[\begin{array}{ll}
0 & 1
\end{array}\right], \quad A_{44}=0 .
\end{gathered}
$$

If we let $L_{\Omega_{1}, \Omega_{2}^{\prime}}=\left[\begin{array}{ll}1 & 0\end{array}\right]$, then we can have $L_{\bar{\Omega}_{1}}=0, L_{\bar{\Omega}_{2}^{\prime}}=$ $\left[\begin{array}{ll}0 & 0 \\ 0 & 1\end{array}\right]$. It follows from Theorem 13 that

$$
\begin{aligned}
\operatorname{rank}\left[\begin{array}{ccc}
L_{\Omega_{1}} A_{11} & L_{\Omega_{1}} A_{13} L_{\Omega_{2}^{\prime}} & L_{\Omega_{1}} A_{14} \\
A_{31} & A_{33} L_{\Omega_{2}^{\prime}} & A_{34} \\
A_{41} & A_{43} L_{\Omega_{2}^{\prime}} & A_{44}
\end{array}\right] \\
=\operatorname{rank}\left[\begin{array}{llll}
0 & 0 & 0 & 0 \\
1 & 0 & 0 & 0 \\
0 & 0 & 0 & 1 \\
0 & 0 & 1 & 0
\end{array}\right]=3=5-\operatorname{rank}\left[\begin{array}{ll}
0 & 0 \\
0 & 1
\end{array}\right]-1
\end{aligned}
$$

implies that there exists a derivative output feedback of the form

$$
u=\left[\begin{array}{ccc}
-1 & 1 & 0 \\
0 & 0 & 0 \\
0 & 0 & 0
\end{array}\right] \dot{y}
$$

such that the resultant closed loop system is impulse free and has dynamical order 2 .

If we choose $L_{\Omega_{1}^{\prime}, \Omega_{2}^{\prime}}=\left[\begin{array}{ll}1 & 0 \\ 0 & 0\end{array}\right]$, which leads to $L_{\bar{\Omega}_{1}^{\prime}}=\left[\begin{array}{ll}0 & 0 \\ 0 & 1\end{array}\right]$, $L_{\bar{\Omega}_{2}^{\prime}}=\left[\begin{array}{ll}0 & 0 \\ 0 & 1\end{array}\right]$, then by applying Theorem 14,

$$
\begin{aligned}
& \operatorname{rank}\left[\begin{array}{cc}
L_{\bar{\Omega}_{1}^{\prime}} A_{33} L_{\bar{\Omega}_{2}^{\prime}} & L_{\bar{\Omega}_{1}^{\prime}} A_{34} \\
A_{43} L_{\bar{\Omega}_{2}^{\prime}} & A_{44}
\end{array}\right] \\
& =\operatorname{rank}\left[\begin{array}{ccc}
0 & 0 & 0 \\
0 & 0 & 1 \\
0 & 1 & 0
\end{array}\right]=2=3-1
\end{aligned}
$$

implies that the derivative output feedback

$$
u=\left[\begin{array}{lll}
0 & 0 & 0 \\
0 & 1 & 0 \\
0 & 0 & 0
\end{array}\right] \dot{y}
$$

can ensure the resultant closed loop system whose dynamical order is assigned to 3 to be impulse free.

Proceeding as in Examples 1 and 2, the parameters of Corollary 3 in [24] can be obtained as

$$
\begin{gathered}
r_{e \gamma}=4, \quad r_{e b}=4, \quad r_{e b \gamma}=6, \quad T_{e b}=\left[\begin{array}{l}
0 \\
0 \\
0 \\
0 \\
1
\end{array}\right], \\
S_{e \gamma}=\left[\begin{array}{l}
0 \\
0 \\
0 \\
0 \\
1
\end{array}\right], \quad \widetilde{T}_{e \gamma}=\widetilde{S}_{e b}=\left[\begin{array}{llll}
0 & 0 & 0 & 0 \\
1 & 0 & 0 & 0 \\
0 & 1 & 0 & 0 \\
0 & 0 & 1 & 0 \\
0 & 0 & 0 & 1
\end{array}\right]
\end{gathered}
$$


from which we have

$$
\begin{gathered}
\operatorname{rank}\left(\widetilde{T}_{e \gamma}^{T} A \widetilde{S}_{e b}\right)=\operatorname{rank}\left[\begin{array}{cccc}
0 & 0 & 0 & 1 \\
1 & 0 & 0 & 0 \\
0 & 0 & 0 & 1 \\
0 & 0 & 1 & 0
\end{array}\right]=3, \\
\operatorname{rank}\left(\widetilde{T}_{e \gamma}^{T} A S_{e \gamma}\right)=\operatorname{rank}\left[\begin{array}{l}
1 \\
0 \\
1 \\
0
\end{array}\right]=1, \\
\operatorname{rank}\left(T_{e b}^{T} A \widetilde{S}_{e b}\right)=\operatorname{rank}\left[\begin{array}{lll}
0 & 0 & 1
\end{array}\right]=1, \\
\operatorname{rank}\left(T_{e b}^{T} A S_{e \gamma}\right)=0 .
\end{gathered}
$$

It follows from Corollary 3 in [24] that the dynamical orders which can be assigned by derivative output feedback are 2 and 3. It can be easily observed that our results coincide with Corollary 3 in [24], regardless of our results being sufficient.

As shown above, derivative output feedback can enable the resultant closed loop system to arrive at any values in the possible range of the dynamical orders, whereas do not always enable it to be impulse free simultaneously; that is, the impulse cannot be completely eliminated by output derivative feedback for all the possibly dynamical orders. In this case, we may incorporate the proportional output feedback which has been studied by many literatures with derivative output feedback to overcome this.

\section{Conclusions}

In this paper, the problem of eliminating the impulsive behavior for descriptor systems by derivative output feedback has been studied. By virtue of a novelly restricted system equivalent form of the original system, the set of possibly dynamical order of the resultant closed loop system by derivative output feedback is characterized; then for different range of this set, some sufficient conditions under which the resultant closed loop system is impulse free via derivative output feedback are presented; moreover, the design methods for derivative output feedback controller are provided. The illustrative examples have been presented to demonstrate the applicability of the proposed approach.

\section{Conflict of Interests}

The authors declare that there is no conflict of interests regarding the publication of this paper.

\section{Acknowledgment}

This work was supported by the National Natural Science Foundation of China under Grant no. 61273008.

\section{References}

[1] Q. L. Zhang, C. Liu, and X. Zhang, Complexity, Analysis and Control of Singular Biological Systems, vol. 421 of Lecture Notes in Control and Information Sciences, Springer, Berlin, Germany, 2012.

[2] D. M. Yang, Q. L. Zhang, and B. Yao, Descriptor Systems, Science Press, Beijing, China, 2003.

[3] Z. Wu, H. Su, and J. Chu, "Delay-dependent robust exponential stability of uncertain singular systems with time delays," International Journal of Innovative Computing, Information and Control, vol. 6, no. 5, pp. 2275-2283, 2010.

[4] Y.-F. Feng, X.-L. Zhu, and Q.-L. Zhang, "An improved $\mathscr{H} \infty$ stabilization condition for singular time-delay systems," International Journal of Innovative Computing, Information and Control, vol. 6, no. 5, pp. 2025-2034, 2010.

[5] Y. F. Teng, Q. L. Zhang, and G. S. Zhang, "Study of Controllability of descriptor systems," Journal of Northeastern University, pp. 1-6, 2003.

[6] Q. L. Zhang and J. Lam, "Robust impulse-eliminating control for descriptor systems," Dynamics of Continuous, Discrete \& Impulsive Systems B, vol. 9, no. 1, pp. 13-27, 2002.

[7] E. Assunção, M. C. M. Teixeira, F. A. Faria, and R. Cardim, "Robust state-derivative feedback LMI-based designs for multivariable linear systems," International Journal of Control, vol. 80, no. 8, pp. 1260-1270, 2007.

[8] T. H. S. Abdelaziz and M. Valášek, "Pole-placement for SISO linear systems by statederivative feedback," IEE Proceedings of Control Theory and Applications, vol. 151, no. 4, pp. 377-385, 2004.

[9] R. Cardim, M. C. M. Teixeira, E. Assuncão, and F. A. Faria, "Control designs for linear systems using state-derivative feedback," in Systems, Structure and Control, pp. 1-28, In-Tech, Vienna, Austria, 2008.

[10] T. H. S. Abdelaziz and M. Valášek, "Direct algorithm for pole placement by state-derivative feedback for multi-input linear systems-nonsingular case," Kybernetika, vol. 41, no. 5, pp. 637660, 2005.

[11] G.-R. Duan and X. Zhang, "Regularizability of linear descriptor systems via output plus partial state derivative feedback," Asian Journal of Control, vol. 5, no. 3, pp. 334-340, 2003.

[12] Y.-C. Kuo, W.-W. Lin, and S.-F. Xu, "Regularization of linear discrete-time periodic descriptor systems by derivative and proportional state feedback," SIAM Journal on Matrix Analysis and Applications, vol. 25, no. 4, pp. 1046-1073, 2004.

[13] D. L. Chu and D. W. C. Ho, "Necessary and sufficient conditions for the output feedback regularization of descriptor systems," IEEE Transactions on Automatic Control, vol. 44, no. 2, pp. 405412, 1999.

[14] F. A. Faria, E. Assunção, M. C. M. Teixeira, R. Cardim, and N. A. P. da Silva, "Robust state-derivative pole placement LMI-based designs for linear systems," International Journal of Control, vol. 82, no. 1, pp. 1-12, 2009.

[15] M. M. Fahmy and H. S. Tantawy, "Dynamical order assignment for linear descriptor systems," International Journal of Control, vol. 52, no. 1, pp. 175-190, 1990.

[16] D. W. Gu, H. P. Petkov, and M. M. Konstantinov, Robust Control Design with Matlab, Springer, Berlin, Germany, 2005.

[17] J. C. Ren and Q. L. Zhang, "Robust normalization and guaranteed cost control for a class of uncertain descriptor systems," Automatica, vol. 48, no. 8, pp. 1693-1697, 2012.

[18] F. A. Faria, E. Assunção, M. C. M. Teixeira, and R. Cardim, "Robust state-derivative feedback LMI-based designs for linear descriptor systems," Mathematical Problems in Engineering, vol. 2010, Article ID 927362, 15 pages, 2010. 
[19] Y.-F. Teng and Q.-L. Zhang, "Stabilization for the closed-loop descriptor system via derivative feedback," Control Theory \& Applications, vol. 25, no. 1, pp. 21-26, 2008.

[20] A. Bunse-Gerstner, V. Mehrmann, and N. K. Nichols, "Regularization of descriptor systems by derivative and proportional state feedback," SIAM Journal on Matrix Analysis and Applications, vol. 13, no. 1, pp. 46-67, 1992.

[21] A. Bunse-Gerstner, V. Mehrmann, and N. K. Nichols, "Output feedback in descriptorsystems," in Linear Algebra for Control Theory, P. van Dooren and B. Wyman, Eds., pp. 43-54, Springer, New York, NY, USA, 1993.

[22] A. Bunse-Gerstner, V. Mehrmann, and N. K. Nichols, "Regularization of descriptor systems by output feedback," IEEE Transactions on Automatic Control, vol. 39, no. 8, pp. 1742-1748, 1994.

[23] D. L. Chu, H. C. Chan, and D. W. C. Ho, "Regularization of singular systems by derivative and proportional output feedback," SIAM Journal on Matrix Analysis and Applications, vol. 19, no. 1, pp. 21-38, 1998.

[24] D. Chu, V. Mehrmann, and N. K. Nichols, "Minimum norm regularization of descriptor systems by mixed output feedback," Linear Algebra and Its Applications, vol. 296, no. 1-3, pp. 39-77, 1999.

[25] Q. L. Zhang, Decentralized Control and Robust Control for LargeScale Descriptor Systems, Northwestern Polytechnic University Press, Xi'an, China, 1997.

[26] R. Marz and R. Riaza, "Linear differential-algebraic equations with properly statedleading term: B-critical points," Preprint 07-9, Institute of Mathematics, Humboldt University, Berlin, Germany, 2007. 


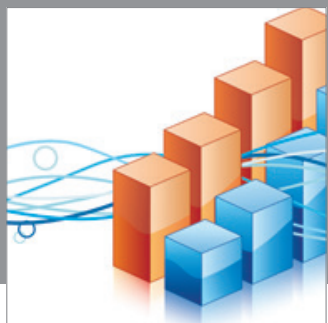

Advances in

Operations Research

mansans

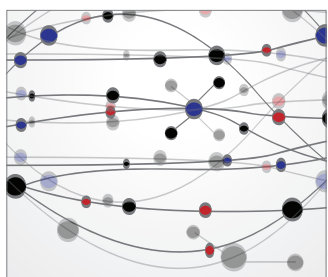

The Scientific World Journal
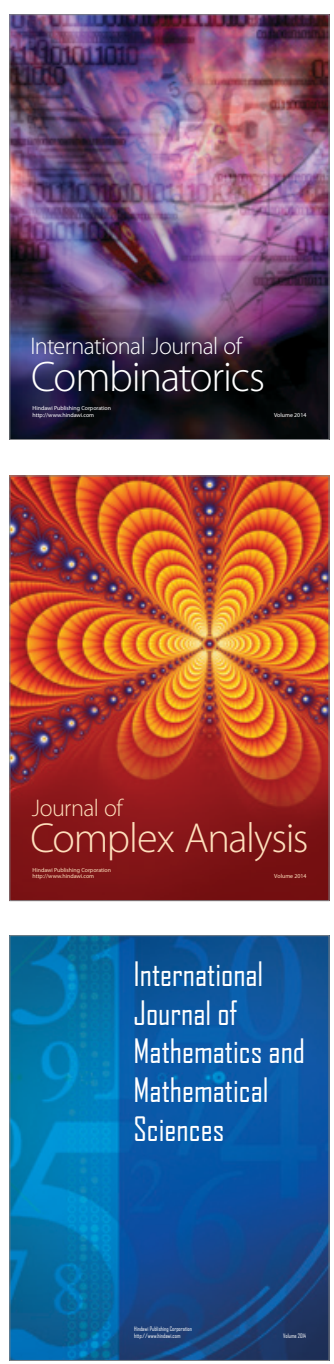
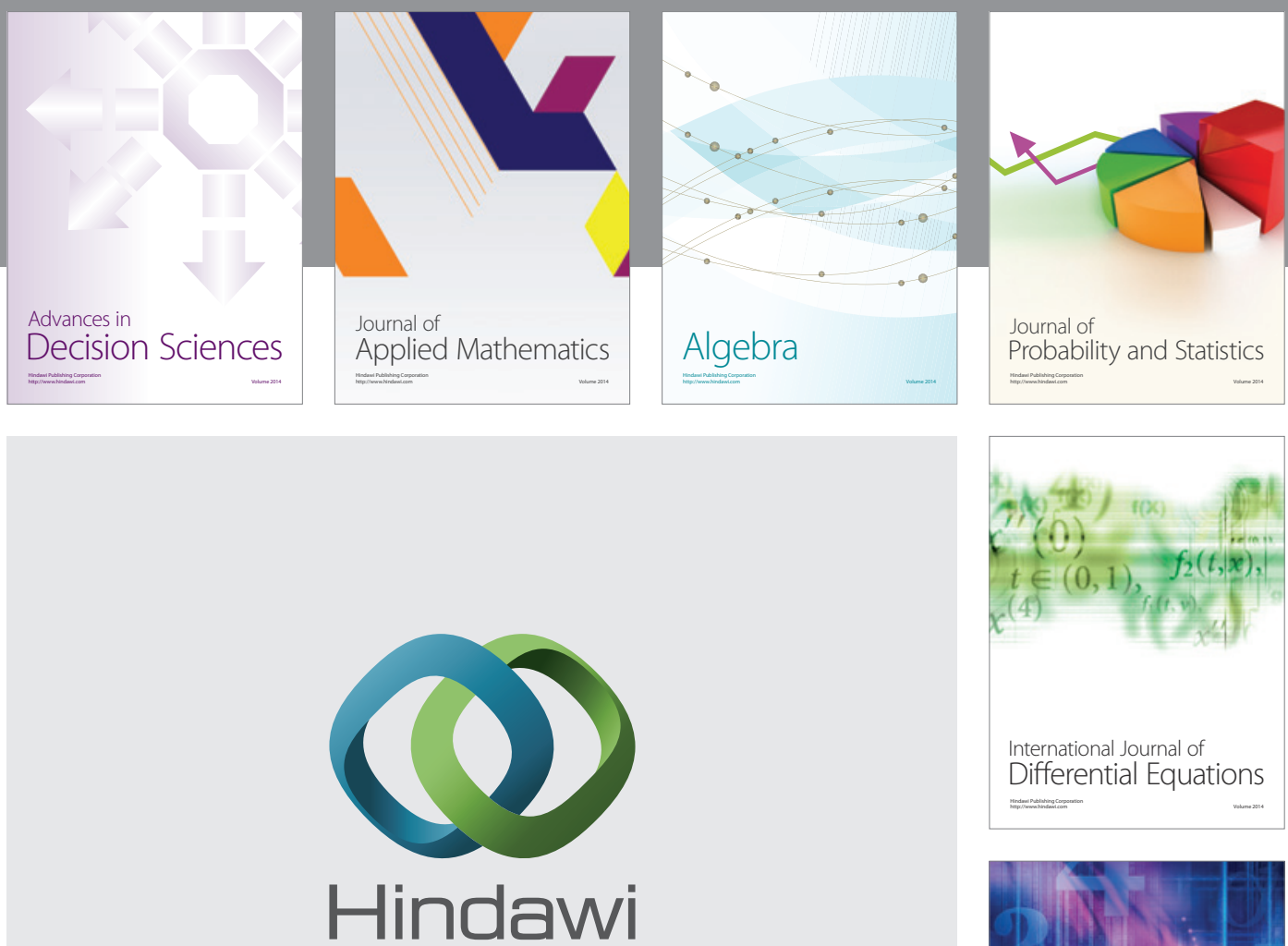

Submit your manuscripts at http://www.hindawi.com
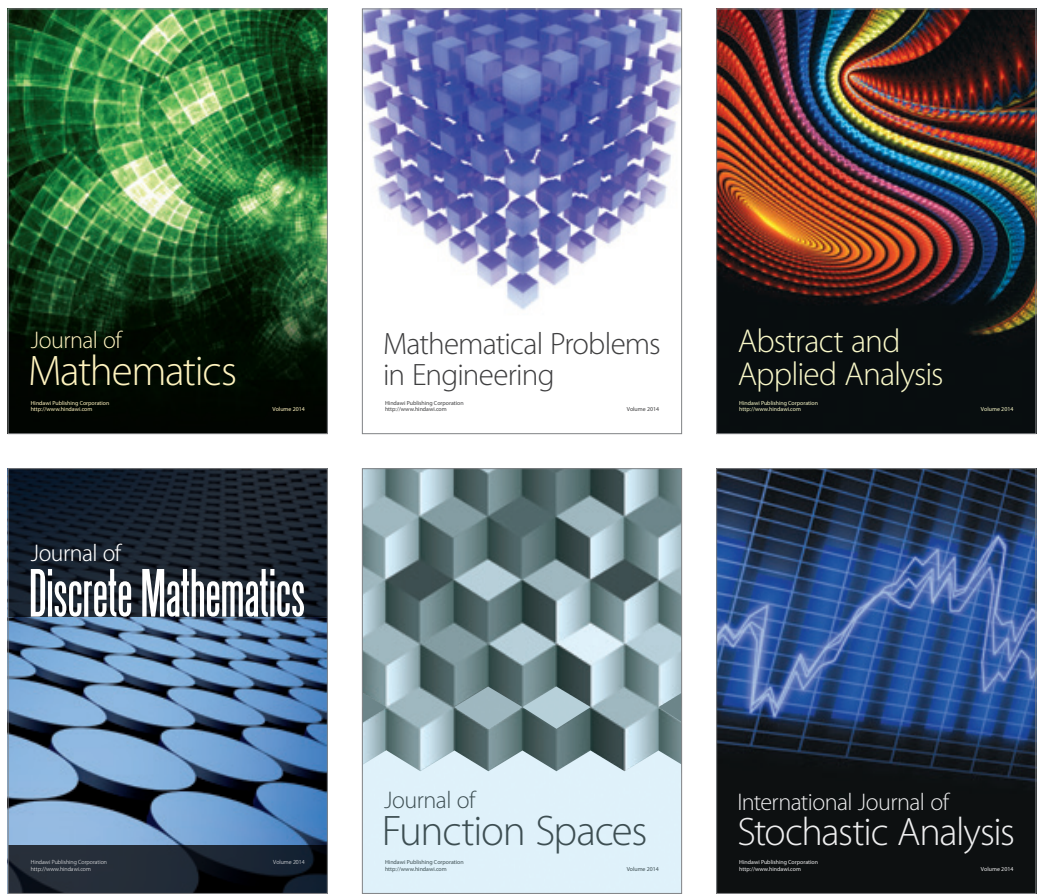

Journal of

Function Spaces

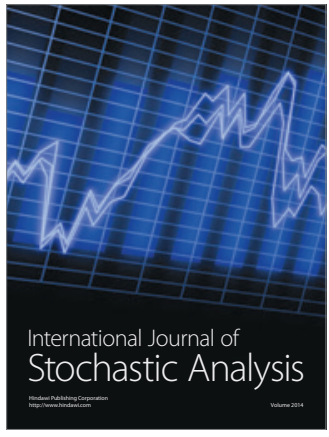

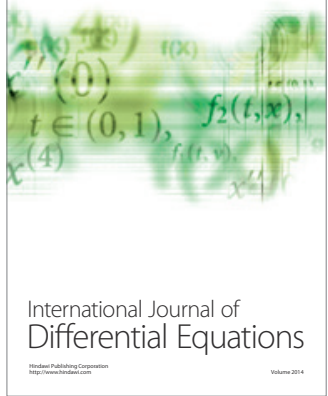
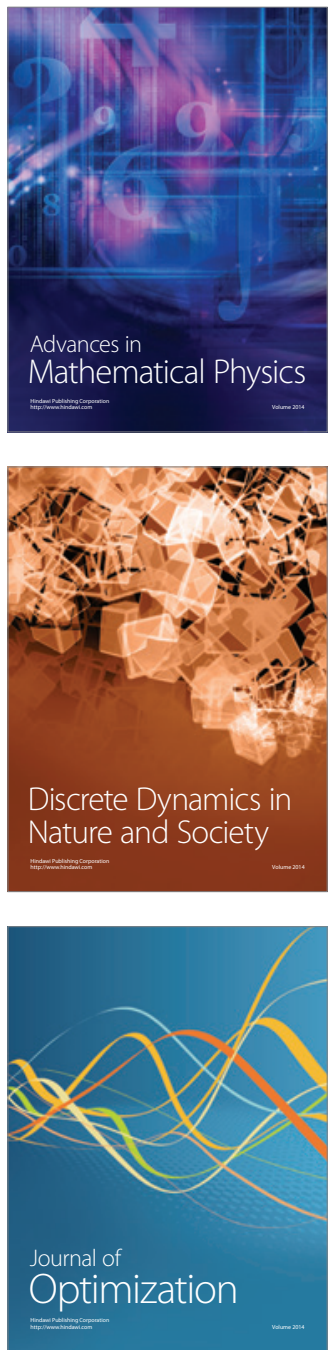